\title{
The MACHO Project: Microlensing Detection Efficiency
}

\author{
C. Alcock ${ }^{1,2}$, R.A. Allsman ${ }^{3}$, D.R. Alves ${ }^{12}$, T.S. Axelrod ${ }^{4}$, A.C. Becker ${ }^{6}$, D.P. Bennett ${ }^{10,1}$, \\ K.H. Cook $^{1,2}$, A.J. Drake ${ }^{1,4}$, K.C. Freeman ${ }^{4}$, M. Geha ${ }^{1}$, K. Griest ${ }^{2,5}$, M.J. Lehner ${ }^{11}$, \\ S.L. Marshall ${ }^{1,2}$, D. Minniti ${ }^{14}$, C.A. Nelson ${ }^{1,2}$, B.A. Peterson ${ }^{4}$, P. Popowski ${ }^{1}$, M.R. Pratt ${ }^{6}$, \\ P.J. Quinn ${ }^{13}$, C.W. Stubbs ${ }^{2,4,6,9}$, W. Sutherland ${ }^{7}$, A.B. Tomaney ${ }^{6}$, T. Vandehei ${ }^{2,5}$, D. Welch ${ }^{8}$
}

(The MACHO Collaboration)

Comments from TV,KG,ML,DA,DB,AB 21 March 2000

\footnotetext{
${ }^{1}$ Lawrence Livermore National Laboratory, Livermore, CA 94550

Email: alcock, adrake, cnelson, dminniti, kcook, mgeha, popowski, stuart@igpp.llnl.gov

${ }^{2}$ Center for Particle Astrophysics, University of California, Berkeley, CA 94720

${ }^{3}$ Supercomputing Facility, Australian National University, Canberra, ACT 0200, Australia

Email: robyn@macho.anu.edu.au

${ }^{4}$ Research School of Astronomy and Astrophysics, Canberra, Weston Creek, ACT 2611, Australia Email: tsa, kcf, peterson@mso.anu.edu.au

${ }^{5}$ Department of Physics, University of California, San Diego, CA 92039

Email: kgriest, vandehei@astrophys.ucsd.edu

${ }^{6}$ Departments of Astronomy and Physics, University of Washington, Seattle, WA 98195

Email: becker, mrp, stubbs@astro.washington.edu

${ }^{7}$ Department of Physics, University of Oxford, Oxford OX1 3RH, U.K.

Email: w.sutherland@physics.ox.ac.uk

${ }^{8}$ McMaster University, Hamilton, Ontario Canada L8S 4M1

Email: welch@physics.mcmaster.ca

${ }^{9}$ Visiting Astronomer, Cerro Tololo Inter-American Observatory

${ }^{10}$ Department of Physics, University of Notre Dame, IN 46556

Email: bennett@bustard.phys.nd.edu

${ }^{11}$ Department of Physics, University of Sheffield, Sheffield S3 7RH, UK

Email: m.lehner@sheffield.ac.uk

${ }^{12}$ Space Telescope Science Institute, 3700 San Martin Dr., Baltimore, MD 21218

Email: alves@stsci.edu

${ }^{13}$ European Southern Observatory, Karl Schwarzchild Str. 2, D-8574 8 Gärching bel München, Germany

Email: pjq@eso.org

${ }^{14}$ Departmento de Astronomia, P. Universidad Católica, Casilla 10 4, Santiago 22, Chile

Email: dante@astro.puc.cl
} 


\begin{abstract}
The MACHO project is a search for dark matter in the form of massive compact halo objects (MACHOs). The project has photometrically monitored tens of millions of stars in the Large Magellanic Cloud (LMC), Small Magellanic Cloud (SMC), and Galactic bulge in search of rare gravitational microlensing events caused by these otherwise invisible objects. In 5.7 years of observations toward the LMC some 13-17 microlensing events have been observed by the MACHO survey, allowing powerful statements to be made about the nature of the dark population in the halo of our Galaxy. A critical component of these statements is an accurate determination of the survey's detection efficiency. The detection efficiency is a complicated function of temporal sampling, stellar crowding (the luminosity function), image quality, photometry, time-series analysis, and criteria used to select the microlensing candidates. Such a complex interdependence is most naturally solved using a Monte Carlo approach. Here we describe the details of the Monte Carlo used to calculate the efficiency presented in the MACHO 5.7-year LMC results. A similar calculation was performed for MACHO's 1-year and 2-year results. Here we correct several shortcomings of these past determinations, including (1) adding fainter source stars (2.5 magnitudes below our faintest detected "stars"), (2) an up-to-date luminosity function for the LMC, (3) better sampling of real images in both stellar density and observing conditions, (4) an improved scheme for adding artificial microlensing onto a random sample of real lightcurves, and many other improvements. The Monte Carlo technique presented here realistically simulates the negative effects of severe crowding (blending) that is a fact of microlensing surveys.
\end{abstract}

Subject headings: dark matter — gravitational lensing — methods: data analysis — Stars: low-mass, brown dwarfs 


\section{INTRODUCTION}

Gravitational microlensing has become an important tool for the discovery and characterization of dark populations. There are now as many microlensing groups Afonso et al. 1999, Alcock et al. 1997a, Ansari et al. 1997, Crotts et al. 1999, Udalski et al. 1997) as there are potential lines of sight out of the Galactic halo (LMC, SMC, Galactic bulge, M31). Surveys toward the LMC have observed an excess in the number of microlensing events over what is expected from known populations of stars. We have recently reported 13-17 microlensing events (Alcock et al. 19999; hereafter A00) in 5.7 years of observations toward the LMC and recently EROSII has reported two new events that they interpret as limiting the amount of halo dark matter (Lasserre et al. 2000), but are consistent with the results of A00. In addition, a number of candidates have been observed towards the SMC (Alcock et al. 1997b, Alcock et al. 1999a, Albrow et al. 1998b, Palanque-Delabrouille et al. 1998, (Afonso et al. 1999, Afonso et al. 1998). One natural explanation for this excess rate is a population of massive compact halo objects of mass $\sim 0.6 M_{\odot}$ that contribute $\sim 20 \%$ to the mass of our Galaxy's halo. However, previously unknown (or underestimated) populations of stellar lenses (e. g., in an LMC halo) are a distinct possibility. In order to make quantitative statements about such a population an accurate determination of the survey's microlensing detection efficiency is required. Here we describe the MA$\mathrm{CHO}$ project's pipeline for calculating its detection efficiency. More details on the experiment, microlensing terminology, analysis, and interpretation can be found in the companion paper A00, and detailed reviews of microlensing in general are given by Roulet \& Mollerach 1997 and Paczyński 1996.

The detection probability for individual events depends on many factors, e.g., the 3 event parameters $A_{\max }, \hat{t}, t_{\max }$ (maximum magnification, Einsteindiameter crossing time and time of peak magnification, respectively), and the unlensed stellar magnitude, as well as the observing strategy and weather conditions. Such a complicated dependence is most naturally solved using a Monte Carlo technique. We may simplify the dependence by averaging over the known distributions in $A_{\max }, t_{\max }$, the stellar magnitudes, and the known time-sampling and weather conditions, to derive owr efficiency as a function only of event timescale, $\mathcal{E}(\hat{t})$.
Given an efficiency $\mathcal{E}(\hat{t})$ we may compute the observed optical depth as,

$$
\tau_{\text {meas }}=\frac{\pi}{4} \frac{1}{E} \sum_{i} \frac{\hat{t}_{i}}{\mathcal{E}\left(\hat{t}_{i}\right)},
$$

where $E$ is the survey's exposure in object-years, $\hat{t}_{i}$ is the duration of event $i$ and $\mathcal{E}(\hat{t})$ is the efficiency for detecting microlensing with duration $\hat{t}$ (see also Alcock et al. 1996 and 1997a; hereafter A96 and A97, respectively). The optical depth is a function of the amount and distribution of mass along the line of sight and is independent of the lens masses or velocities. However, we may extract more information if we assume a model of the Galactic halo. With the use of a model, a likelihood analysis may be performed to determine the most likely lens mass $m$ and mass fraction $f$ of the halo model. A model yields the distribution of event durations $d \Gamma / d \hat{t}$ (Alcock et al. 1995), which, when combined with the efficiency and integrated over all possible durations, predicts the number of events one expects to observe from such a Galactic halo composed entirely of MACHOs.

$$
N_{\text {exp }}=E \int_{0}^{\infty} \mathcal{E}(\hat{t}) \frac{d \Gamma}{d \hat{t}} d \hat{t} .
$$

An identical method may be outlined for stellar populations as in A00. A likelihood estimator may be constructed from the observed events and the modelpredicted number of events to determine simultaneously the most likely lens mass $m$ and mass fraction $f$ of the halo. Note the vital role the efficiency plays in connecting the world of models to that of the survey.

The efficiency is a strong function of the temporal sampling of the survey. Events with very short durations (or very long durations) are unlikely to be detected as they easily fall 'in-between' observations (or for long durations, extend through the data window). A straightforward way of simulating this "sampling efficiency" while retaining realistic behavior of the data is to use a random sample of real lightcurves from the survey in which to inject artificial microlensing. By randomly generating a number of events and running the same time-series analysis and selection criteria used in the real analysis, this Monte Carlo can be used to determine the survey's detection efficiency Alcock et al. 1996, Alcock et al. 1997a, Pratt 1997).

How does one add microlensing onto a lightcurve? A simple method is outlined in $\S 3.2$ for the case where 
one assumes that each lightcurve represents a single resolved star. In this case all of the flux represented by the lightcurve is magnified by the microlensing model $A(t)$ (Equation 4 below). The rarity of microlensing, however, demands crowded stellar fields in order to detect even a handful of events, and this in turn results in the blending of stellar light. The issue now becomes what fraction of the flux in the lightcurve (or object) is lensed? Our solution was first described briefly in A96 (see Pratt 1997 for a more thorough description) and is outlined briefly as follows. The method involves the use of a large set of artificial star tests on a sample of MACHO survey images that are seeded with a color-magnitude diagram of the LMC. Each artificial star was added to an entire series of observing conditions and over 15 peak magnifications. The MACHO survey's dedicated photometry code SoDoPhot was then run on the resulting images to determine how each star behaved over a range of seeing, sky, and magnification. The resulting photometry and photometric flags were stored in binary files, which we refer to as Photometric Response DataBases (PRDBs). In this "photometric efficiency" technique each artificial star (hence forth referred to as a Photometric Response Function or PRF) is used as a model for adding artificial microlensing onto lightcurves. In most cases only a fraction of the observed flux in the lightcurve is actually lensed, and the PRF supplies us this fraction as a function of seeing and sky. In this way both blending and systematic photometry effects are mimicked in the resulting artificial data.

Blending introduces several problems that can affect microlensing surveys in serious ways and has been extensively investigated (Alcock et al. 1996, Wozniak 1997, Pratt 1997, Han 1997, Han 1998). In addition to diluting the true peak magnification $A_{\max }$, blending also biases the measured durations $\hat{t}$ to shorter values, since blended events spend less time above a given threshold. This biasing of $\hat{t}$ is particularly important as the optical depth estimate is proportional to the average duration of the events. Another effect of blending (which has received less attention, but is equally important) is that the survey's exposure in star-years is typically quite a bit larger than is estimated by naively counting photometered objects.

\footnotetext{
1 Blending is when two or more star's point-spread functions overlap to such an extent that the photometry code can no longer identify individual stars, but rather recovers one photometric 'blob' or object.
}

One can view this (for a fixed exposure in objectyears) as a net increase in the efficiency which in part balances out a decrease in efficiency due to blending. All of these effects must be taken into account if one desires an accurate detection efficiency known at least to the level of, if not better than, the shot noise inherent in the low number of events seen toward the Magellanic Clouds.

We make several corrections and improvements to the Monte Carlo presented in A96 and A97. Most notably these are: (1) we add source stars to $V=24.5$, more than 2.5 magnitudes below our faintest detected stars (and 2.5 magnitudes fainter than was used in A96 and A97), (2) we use an up-to-date luminosity function of the LMC, constructed with our groundbased photometry for bright stars $(V<19)$ and HST photometry for dim stars $(V>19)$, (3) we compute luminosity function normalizations separately for each MACHO field, (4) 10 fields of size $5^{\prime} \times 5^{\prime}$ with widely differing stellar density were used to simulate photometry of artificial stars over an average of 69 different observing conditions (only 2 fields with $\sim 20$ observing conditions were used in A96 and A97), (5) an improved scheme for adding artificial microlensing onto real lightcurves, and (6) we describe a robust method of statistically correcting for the $\hat{t}$ bias that can be used to estimate the optical depth.

In $\S 2$ we briefly review the MACHO telescope, photometry system and time-series analysis in order to introduce some MACHO specific vocabulary used throughout the rest of the paper. $\S 3$ outlines the sampling efficiency approach as a primer to the photometric efficiency discussion in $\S$. The results of the photometric efficiency analysis is reserved for $\S$, and we conclude in $\S 6$.

\section{THE MACHO TELESCOPE, PHOTOM- ETRY SYSTEM, AND TIME-SERIES ANAL- YSIS}

The MACHO project has full-time use of the $1.27 \mathrm{~m}$ telescope at Mount Stromlo Observatory, Australia, for a period of 8 years starting July 1992. The telescope was re-commissioned especially for this project, and a computer-controlled pointing and drive system was installed. A system of corrective optics has been installed near the prime focus, giving a focal reduction to $f / 3.9$ with a 1 degree diameter field of view. A dichroic beam splitter and filters provide simultaneous images in two passbands, a "red" band 
$(\sim 590-780 \mathrm{~nm})$ and a "blue" band $(\sim 450-590$ $\mathrm{nm})$. Two large CCD cameras are employed at the two foci; each contains a $2 \times 2$ mosaic of $2048 \times 2048$ pixel Loral CCD imagers. The pixel size is $15 \mu \mathrm{m}$, which corresponds to $0 .^{\prime \prime} 63$ on the sky, giving a sky coverage of $0.7 \times 0.7$ square degrees. Details of the camera system are given by Stubbs et al. (1993) and Marshall et al. (1994) while details of the telescope can be found in Hart et al. (1996).

The survey's photometry code SoDoPhot is a PSF fitting algorithm based on DoPhot (Schechter, Mateo \& Saha 1993). It runs in two different modes. The first mode, a template generation mode, is designed to run on a pair of red and blue images or "chunks." (Each CCD is broken up into 16 roughly $5^{\prime} \times 5^{\prime}$ somewhat overlapping regions called "chunks" for the purpose of photometric reductions.) The red chunk is reduced first in a manner similar to a standard DoPhot reduction. Next the blue chunk is reduced using the results from the red chunk to warmstart the reduction, which improves the star matching between colors. This produces a set of pre-templates for this chunk pair. Once a set of pre-templates for an entire CCD (16 chunks) has been generated the final templates are constructed by enlarging the pretemplates and including stars that lie as much as $\sim 90$ pixels outside the nominal chunk boundaries. This enlargement lessens negative effects on the routine photometry due to incorrect telescope pointing. The final set of template files contains a list of detected objects, their positions (corrected to an airmass of one) and their template magnitudes. A set of template files need be generated only once and is created using images with better than average seeing and dark sky conditions.

All other images are processed in "routine" mode, which proceeds as follows. The appropriate template file is used to warm-start the image by first locating and matching 50 bright unsaturated stars. These stars are used to determine a seven parameter analytic fit to the PSF, a coordinate transformation, and a photometric zero point relative to the template. Then all the template stars are subtracted from the image using the model PSF and coordinate transformation; the variance estimate for each pixel is adjusted to allow for errors in subtraction. Next, photometric fitting is carried out for each star in descending order of brightness, by adding the analytic

\footnotetext{
${ }^{2}$ SoDoPhot $=$ Son Of DoPhot
}

model of the star back to the subtracted frame and fitting a two-parameter fit to the star's flux and sky background, with pixels weighted by inverse variance. The model PSF and computed position of the star are kept fixed. When a star is found to vary significantly from its template magnitude, it and its neighbors undergo a second iteration of fitting. For each star the estimated magnitude and error are determined, along with six other parameters ("flags") measuring (1) the object "type" (single/blended, etc.), (2) the $\chi^{2}$ of the PSF fit, (3) the "crowding," i. e., the amount of flux contributed from nearby stars, (4) the weighted fractions of the PSF masked due to cosmic rays, (5) the weighted fractions of the PSF masked due to bad pixels and (6) the fit sky value.

The set of photometric data points for each detected object are rearranged into a time series and combined with other relevant information (including seeing, average sky brightness, airmass, etc.) into portable binary files called sodsets. The sodsets are in turn passed into our time-series analysis code to search for variable objects and microlensing candidates (see A00 for more details of the analysis). In brief the analysis code calculates for each lightcurve a set of variability statistics, average magnitudes, error bars, crowding, etc., and for lightcurves deemed interesting (level-1 events) a five parameter fit to microlensing is applied, where the parameters are the unmagnified red and blue fluxes, the peak magnification $A_{\text {max }}$, the time of peak magnification $t_{\max }$, and the Einstein-diameter crossing time $\hat{t}$. Many statistics describing the significance of the fit are also stored for the level-1 events.

From the complete set of statistics we designed criteria that select out microlensing candidates from a wide background of noise induced bumps and variable stars. Two different sets of selection criteria (criteria set $\mathrm{A}$ and $\mathrm{B}$ ) were used in $\mathrm{A} 00$, and we refer the interested reader to this paper for full details of the two selection criteria sets, as we give only a qualitative 'feel' for the criteria here. Criteria set A superficially resembles the criteria used in A97 and was designed to be somewhat restrictive in the sense that only good quality, high signal-to-noise $(\mathrm{S} / \mathrm{N})$ events were selected. The criteria are fairly tight and rely strongly on $\mathrm{S} / \mathrm{N}$ statistics that are somewhat microlensingshape specific. Only 13 candidates in the first 5.7 years of LMC data passed criteria set A. In contrast criteria set $\mathrm{B}$ was designed to be inclusive in the sense that any event with a flat baseline and with one sig- 
nificant and unique bump was included. However, criteria set B was somewhat vulnerable to variable stars that exhibit constant baselines for long periods of time with only one outburst, such as exhibited by supernovae, and possibly cataclysmic variables and nova (see A00 for a full discussion on how these interlopers were treated). Some 17 candidates in the first 5.7 years of LMC data passed selection criteria set B.

The relative looseness of criteria set B over A was due to a set of new statistics that allowed us to more accurately characterize and remove the periodic and quasi-peridoic variable star populations. With a major source of background removed the $\mathrm{S} / \mathrm{N}$ level could be lowered. One of the main overall differences in the two sets of selection criteria is that criteria set A is less sensitive on the whole to moderately blended and highly blended events as compared to criteria set B. Also, criteria set B relies less on the microlensingshape information and is less likely to be missing exotic forms of microlensing such as parallax and binary events. Although criteria set B has overall higher detection efficiency than criteria set A the number of detected events compensates and the results presented of A00 are fairly insensitive to the choice of cuts. In what follows we will present both sets of selection criteria and discuss their differences.

\section{THE SAMPLING EFFICIENCY}

\subsection{The One Percent Database of Lightcurves}

A key element in the efficiency determination is the $1 \%$ database of lightcurves. This database represents an unbiased random selection of $1 \%$ of the lightcurves contained in the MACHO project's LMC survey. It is unbiased with respect to observed magnitude, color, sampling rate, spatial distribution, and general data quality. The $1 \%$ database contains 118, 645 lightcurves from the top 30 LMC fields $3^{3}$ and occupies 2.7 Gbytes of disk space. The lightcurves begin on day 200 (= JD $-2,448,623.5 ; 1992$ July 19) and end on day 2267 (1998 March 17) which makes the data window 5.7 years long. The mean number of exposures per field is 719 , with a range from 180 to 1338 . Approximately $13 \%$ of the lightcurves have information in only one passband (red or blue). Of these $\sim 60 \%$ have only red lightcurves and $\sim 40 \%$ have only blue lightcurves. There is a fair amount of field

\footnotetext{
${ }^{3}$ See A00 for a complete list of the 30 LMC fields along with RA
} and dec centers. overlap among the top 30 fields with about $\sim 20 \%$ of lightcurves duplicated across fields.

\subsection{Modifying Lightcurves: Sampling Effi- ciency}

Here we assume that each lightcurve in the $1 \%$ database is a single resolved star, and that our photometry code is "perfect" in recovering flux. We further assume that the measurement errors involved are dominated by photon shot noise. Given a lightcurve we proceed as follows: (1) first a robust mean magnitude $\bar{m}$ for each bandpass is computed. (2) Then a set of event parameters $u_{\min }, t_{\max }$, and $\hat{t}$ is generated where, (a) $u_{\min }$ is chosen as a uniform deviate from zero to the experimental threshold $u_{T}=$ $1.0\left(A_{\max }=1.34\right)$, (b) $t_{\max }$ is chosen as a uniform deviate from slightly before the beginning of the observations to slightly after (day 190 to 2277), and (c) $\hat{t}$ is chosen as a uniform base 10 logarithmic deviate with a duration of 1 day up to 2000 days. (3) For each point on the lightcurve we compute the "scatter" from the mean in flux units as $\Delta f=f-\bar{f}$, where $\bar{f}$ is a robust mean magnitude expressed in flux units, i. e., $\bar{m}=-2.5 \log (\bar{f})$. This scatter is assumed to be due entirely to photometric error rather than intrinsic variation in the source (indeed, only $\sim 1-2 \%$ of all LMC lightcurves show signs of intrinsic variability). (4) The flux $f$ for this point is then "magnified" $f \rightarrow A(u(t)) \bar{f}+\Delta f$, or expressed equivalently in magnitudes, $m \rightarrow \bar{m}-$ $2.5 \log \left(A(u(t))-1+10^{(\bar{m}-m) / 2.5}\right)$. (5) The flux error is modified as $\sigma_{f} \rightarrow \sigma_{f} \sqrt{A(u(t))}$, which translates into magnitude space as $\sigma \rightarrow \sigma / \sqrt{A(u(t))}$. Here $t$ is the time of the observation being modified and $u(t)$ is

$$
u(t)=\left[u_{\min }^{2}+\left(2\left(t-t_{0}\right) / \hat{t}\right)^{2}\right]^{1 / 2},
$$

with

$$
A(u)=\frac{u^{2}+2}{u \sqrt{u^{2}+4}} .
$$

This is performed for all points on the lightcurve and for both bandpasses. We leave the remaining photometric flags $\left(\chi_{p s f}^{2}\right.$, crowding, etc.) unchanged.

\subsection{Calculation of the Efficiency}

Using the technique of $\S 3.2$ to modify lightcurves we proceed to add an event onto each lightcurve in 
the $1 \%$ database. One complete loop through the $1 \%$ database we refer to as a pass. For each "event" we save information such as, (1) the lightcurve id number (field.tile.sequence), (2) a pass number, (3) a robust magnitude $V_{\text {obj }}$ and color $V-R_{\text {obj }}$ of the lightcurve, and (4) the input event parameters $A_{\max }, t_{\max }$, and $\hat{t}$. This bookkeeping information will later be matched with the output of the time-series analysis and used to calculate the efficiency.

Once we have created a complete pass and stored the bookkeeping information we next run the timeseries analysis on the artificial lightcurves ( $\S 2)$. The resulting output statistics and fits are written into a file and then matched on a lightcurve by lightcurve basis with the bookkeeping files that contain the input statistics. Hereafter all parameters labeled with a prime refer to measured parameters (e.g., $A_{\max }^{\prime}$, $t_{\text {max }}^{\prime}$, and $\left.\hat{t}^{\prime}\right)$ and un-primed parameters refer to input or true parameter values. In addition, both criteria sets $\mathrm{A}$ and $\mathrm{B}$ described in $\S 2$ are applied to the resulting statistics, as in A00, and the results stored as an integer: zero $=$ failed the criteria set, one $=$ passed the criteria set. This creates a single datacube file containing input statistics, output statistics, and 'cut' integers. In order to generate enough events to ensure adequate statistics we ran 5 passes through the $1 \%$ database. Though the same lightcurves were used 5 times each, completely different event parameters were generated for each pass. Because we stored a unique pass number in the datacube file we simply concatenated all 5 datacube passes into one large datacube file for ease of use.

With this datacube it is straightforward to calculate the survey's sampling efficiency. Since the artificial events are added with a uniform distribution in $u_{\min }$ and $t_{\max }$, integration over these vari-

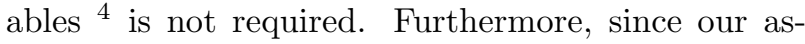
sumption in $\S 3.2$ is that each lightcurve represents a single resolved star, there is also no need to integrate over luminosity (since, under this assumption, the $1 \%$ database fairly samples the survey's luminosity distribution). We need only bin the data in $\hat{t}$ and simply count the number of recovered events versus the number of added events. The efficiency is simply $\mathcal{E}(\hat{t})=N_{\text {rec }}(\hat{t}) / N_{\text {add }}(\hat{t})$.

\footnotetext{
${ }^{4}$ We ignore a small modulation $(\sim 5 \%)$ in the event rate due to the motion of the earth around the sun.
}

\subsection{Results of the Sampling Efficiency}

In Figure 1 we compare the sampling efficiency derived from the 5.7-year data set for both criteria set $\mathrm{A}$ and $\mathrm{B}$ with the previous two data sets, A96 and A97. The general behavior of the efficiency with $\hat{t}$ is easy to understand. The gradual fall in efficiency as $\hat{t}$ decreases is caused by a combination of a typical sampling rate of 1-2 days and large gaps of 3-50 days that exist due to bad weather and other telescope down time. The sharp drop at $\sim 600$ days is due to an explicit cut requiring $\hat{t}<600$ days (affecting both criteria set). The large difference in the efficiencies for the two selection criteria is due to a combination of (1) the different values used for the $A_{\max }$ cut (criteria set A uses $A_{\max }>1.49$ while criteria set B uses $\left.A_{\max }>1.34\right),(2)$ criteria set B uses fewer S/N statistics, most of which are looser, (3) differences in variable star cuts, and (4) various cuts on the minimum quality of the lightcurve (minimum number of points in the red and blue passbands, maximum crowding, etc.).

The most striking difference between the previous two data sets and the 5.7-year set is the much higher efficiency at long durations. Much of this difference is just a reflection of the longer baseline and an increase in the allowed duration $(\hat{t}<600$ days $)$. However, a significant amount of the difference lies in a quirk of the A97 data set. In the A97 data six of the densest fields 5 had their lightcurves cut in half, roughly, due to an early generation of templates used to reduce the photometry for these fields. These early templates made use of a different star naming convention than is currently used. Roughly halfway through the A97 data set the current generation of templates was implemented on these six fields. Since there was no straightforward way to cross reference the ID's of the stars in each different convention (indeed, the map was not even one-to-one), and redoing the photometry was too prohibitive at the time, it was decided to consider as separate lightcurves the stars in these six fields before the upgrade and after the upgrade and to analysis them as such. This was handled in a selfconsistent manner and more details can be found in A97. The primary effect of this was a lowered efficiency for long duration events, due primarily to (1) an effective cut on event peak $t_{\max }$ where the fields were split and (2) the required minimum of 40 baseline points outside $\pm 2 \hat{t}$ of the peak of the event that

\footnotetext{
${ }^{5}$ These fields are $1,7,9,77,78$, and 79
} 
was used in the A97 selection criteria. The problem did not exist in the A96 data, thus the rather close behavior between A96 and A97 for long durations, even though the later had twice the coverage. The A00 data has redone all its photometry in these six fields with the current generation of templates and does not suffer from this problem.

It is also worth noting that in the $\hat{t}$ range 1-40 days the A00 sampling efficiency (for both criteria) is systematically smaller than the A96 and A97 results. In part this is due to differences in the selection criteria, but is also a product of including an additional 8 less densely sampled fields into the year A00 analysis. Though this tends to reduce our efficiency it also increases our exposure. Also note that criteria set B is always above criteria set A except for durations less than 10 days. The slightly lower efficiency of criteria set $\mathrm{B}$ in this range is due to the larger number of required points in the peak $N_{\text {peak }} \geq 10$ (criteria set A only required 7 or more $2 \sigma$ points; see A00).

In addition to $\mathcal{E}(\hat{t})$ it is also interesting to investigate the efficiency as a function of other parameters, such as magnitude, impact parameter and stellar density. Also of considerable interest is the $\hat{t}$ bias mentioned in the introduction. However, we hold off discussing these until after we have introduced the photometric efficiency as this scheme more realistically models the systematics of blending and the survey as a whole.

\section{THE PHOTOMETRIC EFFICIENCY}

In the introduction some of the potential problems associated with the effects of blending were discussed. Blending causes systematic underestimation of both $A_{\max }$ and $\hat{t}$, but also increases the number of stars to which the survey is sensitive. Clearly none of these effects are taken into account by the sampling efficiency described in $\S$ 3. To correct for this deficiency, and to be able to generate realistic microlensing events, we make use of a large database of artificial star photometry as first described in A96 and A97. We have developed techniques that allow us to use this artificial star photometry to inject synthetic microlensing onto $1 \%$ database lightcurves, replacing the techniques described in $\S 3.2$. The new techniques allow us to empirically account for the blending problems, as well as other systematic photometry effects unique to SoDoPhot such as various correlations with seeing, underestimation of error bars and other systematic changes in the photometry flags.

Our method involves a large set of artificial star tests on a sample of the MACHO survey's images that are seeded with a color-magnitude diagram of the LMC. A large set of real images that fairly sample the survey's distributions in observing conditions (seeing and sky) were used to create the database of input photometry versus output photometry for the sample of artificial stars and organized into a PRDB. The PRDB consists of $\sim 60,000$ individual PRFs that represent how a star behaves as part of a blended object. Each star was added to images over a range of observing conditions and over 15 peak magnifications. SoDoPhot was run on the resulting images to determine how each star behaved over the range of seeing, sky, and magnification. The resulting photometry and photometric flags were stored in the PRDBs. Each PRF represents a rule for how a lightcurve would respond to the addition of flux over a wide variety of observing conditions. This can be represented as,

$$
A_{\text {rec }}=A_{\text {rec }}\left(A, V, R, V_{\text {obj }}, R_{\text {obj }} \text {, seeing, sky }\right) \text {, }
$$

where $A_{\text {rec }}$ is the recovered magnification, given an input magnification $A$, stellar magnitudes $V$ and $R$, object magnitudes $V_{\mathrm{obj}}$ and $R_{\mathrm{obj}}$, and characterized by an observing condition (seeing and sky).

To implement the photometric efficiency properly it is important to know the underlying luminosity function (LF) to some limiting magnitude (in our case about $V \sim 24)$. We discuss our determination of the LF in $\S 4.1$. Next, in $\S 4.2$ we describe how the PRDBs are generated, including the images, pointspread functions, coordinate and photometric transformations. In $\S 4.3$ we show some example PRFs and discuss general behaviors exhibited by the PRFs. Discussion of the photometric efficiency (hereafter referred to as just the efficiency) results is left for $\S$.

\subsection{The LMC Luminosity Function and Color- Magnitude Diagram}

It is important to know the LF of stars in the LMC (and to a lesser extent the color-magnitude diagram) in order to accurately estimate the efficiency. This is because the LF, along with stellar density, dictates how much stellar PSF overlap on the sky there is as a function of magnitude and thus how much blending one expects. Also, since the survey's exposure is greater than the number of photometered objects 
suggests, and this can increase the sensitivity of the survey to microlensing, we need a fairly accurate estimate of the number of stars in our fields.

\subsubsection{The Shape of the LMC's Luminosity Function}

The MACHO fields typically go incomplete at magnitudes greater than $V \sim 20$ but can be complete to $V \sim 21$. Because we desire to know the LF to at least $V \sim 24$ we must resort to Hubble Space Telescope (HST) Wide Field Planetary Camera 2 (WFPC2) image data. The $\mathrm{MACHO}$ project has obtained HST

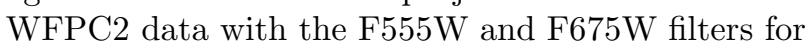
three fields in the LMC bar. These are located in the MACHO fields 2, 11, and 13. For each field, we have obtained "shorts" $(3-4 \times 30 \mathrm{sec}$ exposures $)$ and "longs" (3-4 × 400-500 sec exposures), in both filters. More details of the HST data reduction and analysis are contained in Alcock et al. (1999b). In addition we have obtained the reduced HST WFPC2 data from 6 LMC bar fields obtained and reduced by Olsen 1999 (kindly provided via private communication). These 6 fields are in the F555W and F814W filters and have similar "shorts" and "longs" giving completeness limits similar to our HST data. Olsen's PC was positioned on old LMC globular clusters and so we discarded all PC data and, in addition, must be careful of a small contamination from cluster stars that extend into the edges of the WF CCDs. Details of the Olsen HST reductions can be found in Olsen et al. 1998 and Olsen 1999.

In Figure 2 is plotted the $\mathrm{V}$ band ( $\mathrm{F} 555 \mathrm{~W}$ filter) LF for the 3 MACHO HST fields. Each field is normalized to have the same number of stars in the range $17<V<20 \sqrt{6}$, with the fields of higher stellar density, field 2 and 11, normalized to field 13 (lowest density and lowest $\mathrm{S} / \mathrm{N}$ ). The shape of the 3 LFs appear consistent with one another from $V \sim 17$, where shot noise dominates, to $V \sim 22.5$, where differences in stellar density between the 3 fields cause differences in completeness. Figure 3 is similar to Figure 2 but shows Olsen's 6 fields normalized in a similar fashion to the lowest $\mathrm{S} / \mathrm{N}$ field. Again the shapes of the $6 \mathrm{LFs}$ appear consistent with one another between the shot noise on the bright end and the differing completeness on the dim end.

We can quantify the above 'by eye' assessment by computing a $\chi^{2}$ between fields along with the as-

\footnotetext{
${ }^{6}$ This region corresponds to where the HST fields are greater than $95 \%$ complete.
}

sociated probability of obtaining a value of $\chi^{2} /$ dof worse than the measured value. We find no significant difference between the 3 MACHO LFs with relative measured $\chi^{2}$ /dof of $1.065,1.253$ and 1.242 for the combinations $2-11,2-13$, and 11-13. These correspond to probabilities of $p=0.35,0.11$, and 0.12 , respectively, that the value $\chi^{2} /$ dof could be worse than the measured value. Of the 6 Olsen HST fields one stands out as anomalous in its $\chi^{2} /$ dof value. The LF of NGC 1916 is the most discrepant but appears to be so due to heavy differential reddening (Olsen et al. 1998, Olsen 1999) with relative $\chi^{2} /$ dof ranging from a low of 1.589 to a high of 3.777 , corresponding to a range of probabilities $p=5.3 \times 10^{-3}$ to $1.1 \times 10^{-17}$. We thus discard this field from further analysis. The remaining 5 Olsen LFs are consistent with one another, with relative $\chi^{2} /$ dof ranging from a low of 1.118 to a high of 1.891 , corresponding to a range of probabilities $p=0.26$ to $1.6 \times 10^{-4}$. The slightly worse probabilities seen in the Olsen fields are likely due to contamination from the globular clusters spilling over into the WF CCDs.

In Figure 4 we plot MACHO's combined LF (3 fields) and Olsen's combined LF (5 fields), normalized to the MACHO combined LF in the range $17<$ $V<20$. The two LFs are consistent with one another in the range $17<V<22\left(\chi^{2} /\right.$ dof $=1.215$, $p=0.17)$. Also plotted is MACHO's ground-based LF for field 13 ( 350, 000 objects) which is one of our most complete, lowest reddened fields, with excellent template seeing and sky (we have allowed for a small offset of $\sim 0.1$ in $V$, see below). The shape of the ground-based LF in the range $17<V<21$ is good in comparison with the MACHO combined HST LF $\left(\chi^{2} /\right.$ dof $\left.=1.295, p=0.10\right)$. Given the apparent lack of any significant difference in the shapes of the LFs for these 9 LMC bar fields we have chosen to combine 8 of them (excluding NGC 1916 because of high reddening) to form a LF with a fairly high $\mathrm{S} / \mathrm{N}$ on the $\operatorname{dim}$ end (to $V \sim 22.5$ ) and to splice this together with the bright end $(V<20)$ of the MACHO ground-based LF. In this way we create a high $\mathrm{S} / \mathrm{N}$ $\mathrm{LF}$ that is complete in the range $15<V<22.5$.

There is still a question of the completeness of the HST LFs for magnitudes greater than $V \sim 22.5$. We have performed artificial stars tests on our $3 \mathrm{MA}-$ CHO HST fields and used the resulting completeness curves to correct the MACHO LF to $V \sim 24.5$. The completeness tests probably represent a slight underestimate of the true LF. Because of the poten- 
tial uncertainty in the shape of the LF for dim stars we have opted to create two different LFs in which to test for systematics. These are shown in panel (a) of Figure 11. The first LF (hereafter $L F_{1}$ ) has been extended from $V \sim 22$ to greater magnitudes using a power law with a slope of 0.415 (Alcock et al. 199 b) derived from a linear regression fit between $19.5<V<22$. This LF probably represents an overestimate of the number of faint stars. The second LF (hereafter $L F_{2}$ ) uses the completeness corrected MACHO LF as an estimate of the shape for dim stars. The shape of the true LF is likely to lie somewhere in between these two, but is probably closer to the completeness corrected $L F_{2}$. We choose to use both LFs in order to estimate any systematic error induced by such an uncertainty.

\subsubsection{Normalization to MACHO Fields}

With the shape of the LF determined it only remains to find a normalization, that is, a number relating these two LFs to the total number of stars. This is most convenient to do on a field by field basis as the stellar density changes quite rapidly across our fields (and also inside our fields, but we average over this). A normalization is calculated for each field using the ground-based LF for that field and the 'universal' LFs shown in panel (a) of Figure 11. This is illustrated in Figure 5 where four MACHO fields of widely differing stellar density are shown.

First, we allow a slight offset in $\mathrm{V}$ magnitude that is field dependent and typically varies from $-0.14 \mathrm{mag}$ to $0.40 \mathrm{mag}$ (Table $\mathbb{1}$ ). This slight offset is due to a combination of three effects, (1) extinction (patchy, even on the scale of our fields), (2) the tilt of the LMC's disk (maximum effect \pm 0.07 in our 30 fields), and (3) fields with poorly calibrated photometry. We note that the offsets are not correlated with seeing, sky, airmass or stellar density of the template images, but are strongly correlated with the color of the LMC clump, indicating that differences in extinction are

\footnotetext{
${ }^{7}$ We warn the reader that the LF in the bar appears to be 'universal', and we use this simplifying assumption for the present results. The HST fields used here only represent the LF in the LMC's bar, while the LMC's disk LF has been shown to be somewhat different in that it lacks an intermediate age population (Geha et al. 1998, Olsen 1999). Our outer most fields 53, 55 and 57 are farther out in the disk and could thus be in error, though we note that NGC 1754 is also in the disk and, although it shows a different star formation history than the bar. apparently its LF is still fairly similar to the bar fields (Olsen 1999).
}

the dominate cause. The offsets are derived by requiring the peaks of the clump and the tips of the giant branch to line up in $V$ with the 'universal' LFs. The effect of extinction is to lower the number of source stars to which the survey is sensitive. Only $22 \mathrm{LMC}$ fields are well-calibrated (Alcock et al. 1999b) and these typically have the smallest offsets, while 8 LMC fields have only approximate calibrations and typically have large positive offsets. Once the offsets are applied we next require the number stars in the "universal' LFs in the range $17.5<V<18.5^{\text {月 }}$ to match the number of objects in the ground-based LF in the same range. The two shifts typically align the 'universal' LF to the observed LF quite well, both inside and outside the calibration bin, as seen in Figure 5 .

Figure 6 displays the normalizations for each of the 30 MACHO fields derived in the manner described above. Our choice of units for the normalization is the ratio of the number of stars to the number of objects brighter than magnitude $V$. In this case $V=22$ is convenient as there are few objects dimmer than this limit. The $S / O(V<22)$ ratios are plotted versus the average density (in objects $/ \square^{\prime}$ ) for each field as solid circles and labeled with the field number. The normalizations are also supplied in Table1. Note the general trend of increasing $S / O(V<22)$ with increasing object density, which is simply a reflection of the fact that our fields become less complete in more crowded fields. The substantial amount of scatter seen at any given density is a reflection of other factors affecting completeness. For example, $S / O(V<22)$ is strongly correlated with template sky for any given density, in the sense that fields with high sky tend to be less complete (have higher $S / O(V<22)$ ). Though $S / O(V<22)$ appears not to be significantly correlated with template seeing this is not surprising as the range in seeing for our templates is not nearly as large as the range in sky.

Using our 3 HST fields we may make a direct determination of $S / O(V<22)$ by simply counting the MACHO objects that lie inside the WF CCDs. These are shown in Figure 6 as solid triangles. We have connected points from the same fields with a solid line. Note that the density shown for the field $S / O(V<22)$ normalizations is an average over the whole field, while for the HST $S / O(V<22)$ normalizations it is only over the WF CCDs. In all three

\footnotetext{
${ }^{8}$ This region corresponds to where the MACHO ground-based fields are greater than $95 \%$ complete.
} 
cases the HST frames are in higher than average density regions of the MACHO fields, resulting in higher $S / O(V<22)$ ratios. The overall agreement between $S / O(V<22)$ as determined using the individual field normalizations and as determined directly with HST is reassuring.

We have also created three synthetic images as an additional check on the normalizations and to look for possible biases due to blending. Briefly, the synthetic images are $5^{\prime} \times 5^{\prime}$ in size and were generated using empirical PSFs (see $\S 4.2$ ) derived from the template images. A LF similar to $L F_{1}$ above was used to add $\sim 500,000$ stars down to $V=24$ uniformly over the image. A uniform sky with Poisson noise was added to match the ADU/pixel distribution and then SoDoPhot was run in template generation mode on these synthetic images. The resulting number of recovered objects and sky were compared with the real image values simulated. Several iterations over the number of added stars versus added uniform sky were required to match the observed number of objects and sky level. The $S / O(V<22)$ ratio for each of these synthetic images is shown as an open triangle in Figure 6 and is connected by a solid line to its corresponding field average. The densities of the three synthetic images are in general different from the corresponding field averages, based on where the chunk lies in the field. Note the very similar behavior of the synthetically derived $S / O(V<22)$ compared with the field average $S / O(V<22)$ values and the HST derived $S / O(V<22)$. In all three cases the higher density had the higher $S / O(V<22)$ ratio. Also note the much larger increase in $S / O(V<22)$ for the higher densities. This suggests a saturation point is reached in our densest fields whereby the addition of more stars results in a dwindling increase in the number of detected objects as the stars pile on top of one another in the image. We see a similar behavior in the synthetic images where to recover a given number of objects one must add a proportionally larger number of stars.

Using the synthetic images we may also estimate the size of a possible bias in the normalizations due to blending. It would be desirable for our normalization bin $(17.5<V<18.5)$ to contain an equal number of photometered objects and real stars: i. e., $N_{\text {objects }}(17.5<V<18.5) / N_{\text {stars }}(17.5<V<18.5)=$ 1.0. Our synthetic images have values of $1.04 \pm 0.08$, $1.04 \pm 0.05$, and $1.18 \pm 0.04$, where the errors are $1 \sigma$ Poisson errors. They all appear to have slightly more pho- tometered objects than real stars in the normalization bin, though two are entirely consistent with 1.0. Only the highest density image has a value that is significantly larger than 1.0 (but by $4.5 \sigma$ ). This has the potential of biasing our derived values of $S / O(V<22)$ to larger values. However, of the three synthetic images two have extreme densities (as these were chosen to look for this effect) and are not representative of the average density in our fields (see Figure 6). Because we expect the biases to be strongest in our most crowded fields (and to a lesser extent worse seeing template images) the overall bias is certainly much less than the $18 \%$ seen in the extreme artificial chunk, and probably even less than the straight average of $\sim 9 \%$. Another estimate ${ }^{9}$ puts the possible bias at 6 $8 \%$. Given the fairly small size of the effect $(<10 \%)$ we make no correction for this bias, but include it in our error budget (see $\S 5.3$ ).

The errors in $S / O$ (Table 1 ) were estimated as follows: (1) we allowed an uncertainty in the offsets of $0.1 \mathrm{mag}$, based on the scatter in the offsets between fields, and (2) based on the estimates of the maximum blending bias discussed above, the normalizations could be at most $10 \%$ too high and probably only $5 \%$ too low. We then propagated these two uncertainties through the normalization procedure to produce the final errors in the table. The average error in $S / O$ per field is $\sim 20 \%$. The density (objects $/ \square^{\prime}$ ) weighted mean number of stars to objects with $V<24$ is $\overline{S / O(V<24)}=17.05 \pm 3.0$ for $L F_{1}$ and $\overline{S / O(V<24)}=10.83 \pm 1.9$ for $L F_{2}$. The normalization of each field amounts to correcting the MACHO nominal exposure in object-years to an exposure in star-years. The uncertainty in the normalization is moderately large and translates directly into a moderately large uncertainty in the efficiency as discussed in $\S$.

\subsubsection{LMC Color-Magnitude Diagram}

Since we simultaneously image in both a red and a blue passband we require a color-magnitude diagram (CMD) from which to draw our artificial stars. Such a CMD was created for precisely this purpose and its construction is described in detail in Alcock et al. (1999b). Briefly, we spliced the bright end $(V<18.7)$

\footnotetext{
${ }^{9}$ This second estimate uses a simple physical model of blending that fits a model through the three values of $N_{\text {objects }}(17.5<$ $V<18.5) / N_{\text {stars }}(17.5<V<18.5)$ versus $N_{\text {stars }}(17.5<V<$ $18.5)(\text { seeing })^{2}$ and extrapolates the results to all 30 fields. A net bias of $6-8 \%$ is estimated.
} 
of a CMD derived from $\sim 9$ million objects Alcock $e$ al. 1999d from our 22 calibrated ground-based fields onto the dim end $(V>18.7)$ of a CMD derived from our combined 3 HST fields (which is scaled by relative sky area to the $9 \mathrm{M}$ CMD). The HST CMD was corrected on the $\operatorname{dim}$ end $(V>22)$ using a completeness function that corrected the data to a slope of 0.415 in $\log (d N / d V)$ versus $V$. A small amount of editing was also required to remove isolated high pixels (due to the scaling up of the lower S/N HST data) and bright foreground stars. The CMD used here need not be accurate (though we endeavored to make it so) as its only purpose is to draw artificial stars from a realistic distribution in color. We expect no dependence of the efficiency on color (and see none) and so henceforth discard any color dependency.

\subsection{The Photometric Response DataBase}

To create the photometric response database we selected 10 sub-images, approximately $5^{\prime} \times 5^{\prime}$ in size ("chunks"), that span the range of stellar densities observed in the 30 MACHO fields $(\sim 93$ to $\sim 317$ objects $/ \square^{\prime}$; see Table 11) and that lie at a range of distances from the optical center of the bar (from 10 arcmins to 3.2 degrees). For each of these 10 chunks we extracted from the MACHO archive a range of observing conditions that fairly sample the survey's distribution in seeing and sky. Table 2 lists some relevant parameters for each chunk, including MACHO field and chunk id, template observation number, density in objects $/ \square^{\prime}$, number of observing conditions $N_{\text {cond }}$, number of stars per grid $N_{P R F} /$ grid (see below), and the total number of useful PRFs. The mean number of observing conditions for these 10 chunks is 69 , a factor of 3 more than used in A96 and A97.

In order to add artificial stars to these images we require knowledge of the point-spread function (PSF) for each image. We also need a photometric and coordinate transformation (CTR) that maps the photometry and position of the artificial stars to some reference image, in this case the template images for each chunk. We generated empirical PSFs using a PERL script that automates the astronomical photometry package DaoPhot/AllStar (Stetson 1992). The script iterates multiple times with a series of simple analytic PSFs searching for stars, fitting, and subtracting neighbors to candidate PSF stars on each iteration while using a progressively more realistic model of the PSF. The final PSF is a Lorentz-Gaussian analytic model with an empirical look-up table of corrections.
PSFs for each of the $\sim 1600$ chunks are generated in this fashion, and are of higher quality than the simple modified Gaussian analytic model used by SoDoPhot. We visually inspected a number of PSF subtracted images and were quite satisfied with the subtraction. AllStar photometry, using these PSFs, was run on all $\sim 1600$ chunks and the results fed into a custom code that searches for and computes both photometric and coordinate transformations. The code is based on the Groth (1986) algorithm and incorporates a color dependent term in both photometric and coordinate transformations. The inclusion of color in the transformations allows for the effects of airmass, differential refraction, and a known systematic pier side CCD effect in the MACHO data ("blue jitter"; see Alcock et al. (1999b)). From the residuals to the fits we find the coordinate transformations are good to $\sim 0.18$ pixels and the photometric transformations to $\sim 0.08 \mathrm{mag}$.

Grids of artificial stars are added to each of the 10 chunks over all $\sim 69$ observing conditions using the appropriate PSFs and CTRs for each observing condition. The grids contain from 64 to 156 artificial stars (Table 2) positioned on a pseudo-random spatial grid such that stars are never closer than $\sim 21$ arcsecs (or approximately $\sim 7$ FWHM in median seeing) and never closer than $\sim 20$ arcsecs to a boundary of the chunk. This is to avoid missing too much data because of telescope pointing errors. The magnitudes of the artificial stars, $V$ and $R$, are drawn from the CMD of $\S 4.1 .3$ in the range $16.5<V<24.5$ and $-0.5<V-R<1.5$. The CMD is sampled in a square-root fashion to ensure uniform statistics over the large range in luminosity. Each star in the grid is added over a range of 15 peak magnifications $A=$ $0.8,1.0,1.1,1.2,1.34,1.5,2,3,4,5,10,20,30,40,50$. In each image the peak magnifications for each star are staggered so as not to add too many highly magnified stars to a given image. To cover as much of each chunk's image plane as possible, 60 different grids are created for each chunk. This corresponds to covering $\sim 30-70 \%$ of the image plane (depending on the chunk) and represents a statistically significant number of possible photometric conditions in which a star could reside.

All stars in a grid are first added with a magnification of one to the template image of the chunk and SoDoPhot is run in template generation mode on these template images to create the template files. With the template files created, we next loop through 
all observing conditions and all possible peak magnifications, adding the grid to the corresponding observing condition using the appropriate PSFs, CTRs, CCD gains, and with a staggered magnification for each star. SoDoPhot in routine mode is run on each image created and the results are organized and stored in PRDB files. We chose to store all photometry on the three spatially nearest recovered objects to each artificial star in order to map out where the added flux goes. For each grid of stars a PRDB file with 17.4 Mbytes of photometry is created. In total the PRDB contains 10.4 Gbytes of photometry on 196,740 recovered objects.

The total number of artificial stars added to all 10 chunks over the 60 grids per chunk is 65,580 . Of these 54,981 artificial stars contain enough reduced data (greater than $50 \%$ in each passbands) to be useful as PRFs. This is a factor of five more PRFs than was used in A96 and A97. Approximately $16 \%$ of the PRFs were lost due to a combination of (1) falling inside an obliterated region caused by a saturated star $(\sim 2 \%),(2)$ missing data entirely in either the red $(\sim 3 \%)$ or blue $(\sim 6 \%)$ bandpass, and (3) falling near an obliterated region, CCD defect, or chunk edge such that on average more than $50 \%$ of the photometry was missing $(\sim 5 \%)$. Since we are only concerned with how SoDoPhot responds to added flux the loss of poorly determined PRFs is not a concern. The number of single bandpass lightcurves, CCD defects, and other missing data are preserved in the $1 \%$ database of lightcurves.

\subsection{The Photometric Response Functions}

Initially we were concerned with how SoDoPhot divides up flux between two or more closely spaced stars, and which neighbor is most 'sensitive' to the added flux. For example, in A00 two lightcurves (events $7 \mathrm{a}$ and $7 \mathrm{~b}$ ) are, in fact, the same event. Event 7 was bright enough and in a locally crowded enough region that some of the flux from the primary (7a) bled into a secondary neighbor (7b) causing a spurious detection. Although event $7 \mathrm{~b}$ was removed from the list of microlensing events and had no adverse effect on the results of A00, it underscores the need to investigate what effect multiple crowded neighbors have on our efficiency. To investigate this we stored the photometry for the three spatially nearest objects to each artificial star in the PRDBs. We found that in the vast majority of cases $(\sim 97 \%)$ the nearest neighbor snatched most of the added flux and was the most 'sensitive'. This is reassuring, both for simplicity's sake and because our previous work (A96 and A97) had implicitly assumed this behavior. We illustrate this as follows.

For each neighbor we define a recovered magnification, $A_{\text {rec }}(i, j)=f(i, j) / \bar{f}$, where $f(i, j)$ is the flux of the neighbor for observing condition $i$ and peak magnification $j$. Here $\bar{f}$ is the baseline flux for the neighbor (that is the flux averaged over all $i$ with $j=0$ corresponding to $A=1.0$ ). A scatter plot of $A_{\text {rec }}$ versus $A$ for all $i$ shows an excellent linear relationship as expected (see below), with a fit slope $m=d A_{r e c} / d A$ that is generally between 0 and 1 . This slope corresponds to the average blend fraction. We constructed a measure of sensitivity to added flux and computed this measure for each of the three neighbors to each artificial star. The measure we used was the fit slope $m$ divided by a mean relative error bar $\sigma$ for the baseline, i. e., measure $=m / \sigma$. This measure ensured that neighbors with large slopes and small error bars are counted as the most sensitive, while in the case of two neighbors with equal slopes the neighbor with the smallest error bars is counted as the most sensitive. This is likely to be the case as both selection criteria set A and B make use of a signal-to-noise cut requiring the magnification to exceed some multiple of the mean relative error bar (see $\S$ 2 2 and A00).

For each artificial star, the three nearest neighbors are ranked according to the measure, and a cut $A_{\max }>1.34$ was applied to each neighbor to ensure that the PRF was not 'junk' (here $A_{\max }$ is the maximum magnification of the recovered neighbor). Only in $\sim 3 \%$ of the cases was either the 2 nd or 3 rd nearest neighbor more 'sensitive' than the nearest neighbor. We visually inspected a large number of these cases. In most cases there were two neighbors about equally spaced from the artificial star with neighbor 1 (the closest) dimmer than neighbor 2. Neighbor 2 was more 'sensitive' for two reasons, (1) being brighter it had the smaller relative error bar and (2) due to a slight systematic bias in SoDoPhot the brighter stars, which are reduced first, can pirate flux from the wings of nearby dim stars. Since only $\sim 3 \%$ of cases are in error due to this effect, we restrict our attention to only the nearest neighbor.

A scatter plot of $A_{\text {rec }}$ versus $A$ over all observing conditions is shown for four PRFs in Figure 7 . Note the excellent linear relationship fit by the solid line. The dashed line is the simple analytic response function used in the sampling efficiency $(\S 3.2)$ and is 
plotted here for comparison. The panels correspond to (a) a unblended PRF $(m=1.0)$, (b) a somewhat blended PRF $(m=0.77)$, (c) a moderately blended PRF $(m=0.65)$, and (d) a heavily blended PRF $(m=0.35)$. The scatter at each $A$ is composed of $\sim 69$ observing conditions and is typically well correlated with seeing in the sense that worse seeing induces slightly larger magnifications. This is easy to understand, since worse seeing implies the PSFs overlap more and thus more flux can be contributed to the nearest neighbor by a magnified star. A few cases of very high positive correlation and even negative correlation exist and correspond to cases where (1) the artificial star is not directly detected and lies a moderate distance from the nearest neighbor so that when it is magnified it only affects its nearest neighbor in poor seeing and (2) the artificial star lies almost equidistant between the two nearest neighbors and in good seeing contributes to the nearest neighbor but in bad seeing contributes more to the second nearest neighbor.

To investigate how the relative error bars $\sigma$ (error bars expressed in magnitudes) behave versus $A$ we define an effective error 'de-magnification' as $A_{\sigma}(i, j)=$ $\sigma(i, j) / \sigma(i, j=0)$, where $\sigma(i, j)$ is $\sigma$ for observing condition $i$ and peak magnification $j$. We normalize the error bar magnification separately for each observing condition $i$ because $\sigma$ is in general highly correlated with seeing (becoming larger in worse seeing). Notice that $A_{\sigma}(i, j) \rightarrow 1.0$ for all $i$ when $j \rightarrow 0$ (that is $A \rightarrow 1.0$ ). Figure 8 is a scatter plot of $A_{\sigma}$ versus $A$ for the same set of PRFs as shown in Figure 7. Over-plotted as a solid line on each panel is the purely Poisson behavior used in the sampling efficiency $\left.\left(A_{\sigma}=1.0 / \sqrt{(} A\right)\right)$. The behaviors of the PRF's relative error bars show little resemblance to this purely Poisson approximation. Rather the PRFs behave more like $A_{\sigma}=1.0 / A$ (dashed line in the figure), which can be understood as follows.

In our most common case the noise is dominated by a combination of the Poisson noise in the sky plus extra Poisson noise added due to neighboring stars which have been subtracted during the photometry reductions. These are both independent of magnification, so the error in flux should not depend on magnification. That is, the error in linear flux units should not change at all, while the relative errors should behave as $\sim 1.0 /$ flux or, as in our case $\sim 1.0 / A$. This is the pattern we see in our PRFs. However, very bright stars typically fall into the purely Poisson limit. Heav- ily blended PRFs can also approach the Poisson limit, and in some cases their relative error bars change very little as the magnified artificial star is only a small perturbation on the much brighter neighbor.

The broad range in behavior of the PRFs is illustrated in Figure 9, which is a scatter plot of fit slope $m=d A_{\text {rec }} / d A$ versus magnitude $V$ for $\sim 59,000$ PRFs. The amount and distribution of blending is immediately apparent in this plot, with the artificial stars being strongly bifurcated towards either being recovered within $\pm 10 \%$ of their input flux or being blended by greater than $\sim 90 \%$. However, there are still a substantial number that are recovered at intermediate blend fractions, and as the artificial stars shown here represents a square-root sampling of the CMD (thus under-weighting dim stars; see $\S 4.2$ the corresponding results for a linear sampling would show proportionally even more blending. The left ordinate is the fit slope $m$ and the right ordinate is scaled to the maximum recovered magnification that each PRF contains (due to our maximum input magnification of 50). For example, an artificial star with $m=0.5$ would contain a maximum recovered magnification of $A_{r e c}=25$ in the PRF (with any higher magnifications needing to be extrapolated beyond this point, see $\S 4.4$.

Also plotted in Figure 9 is a family of five smooth curves that correspond to model $m(V)$. Each curve in the family assumes a certain amount of blended flux corresponding to $V_{\text {blend }}=18,19,20,21$, and 22 mags in the figure. For example, a star blended with 20 mags of additional flux (the center curve) would fall at $m=0.7$ if it was $V=19, m=0.5$ if it was $V=20$, and $m=0.25$ if it was $V=21$. Note how the family of curves brackets the scattered points fairly well. The solid horizontal line labeled $A=1.75$ illustrates that stars fainter than $V \sim 24$ rarely, if ever, are magnified greater than this limit. As a consequence, if one desired to add stars fainter than $V \sim 24$, one would also need to add them at magnifications greater than 50 .

The PRFs also allow us to model how the photometry flags (crowding, $\chi_{P S F}^{2}$, fit sky, etc.) are handled by SoDoPhot under various observing conditions and peak magnifications. We briefly summarize the noteworthy effects here. The crowding parameter, which is a measure of the amount of contaminating flux from nearby neighbors, not surprisingly, is highly correlated with seeing. As such we define a multiplicative 'magnification' in a fashion identical to the relative error bars discussed above. In most cases ( $\gtrsim 80 \%)$ there 
is little or no variation of crowding with $A$. However, in a few cases the crowding parameter increases smoothly with $A$. The $\chi_{P S F}^{2}$ is also strongly correlated with seeing, but in the sense that the fit to the PSF is worse in better seeing. This anti-correlation is mostly due to the photometered object being made up of multiple overlapping PSFs, which are smoothed out in bad seeing. Unlike the crowding parameter, the $\chi_{P S F}^{2}$ is anti-correlated with $A$, in the sense that high magnifications result in poor fits to the PSF. The fit sky parameter is not significantly correlated with either seeing or sky, and in only a few cases $(\lesssim 10 \%)$ is the fit sky well correlated with $A$, usually in the sense that it is higher for larger peak magnifications, but not always. Neither the missing pixel or cosmic ray flags significantly correlate with seeing, sky, or magnification. The object type is more complicated, but fortunately for our purposes is not important. How the object type changes with seeing and sky is already properly handled in the $1 \%$ database and how it might change with magnification is inconsequential as long as it remains a valid type.

\subsection{Modifying Lightcurves: Photometric Ef- ficiency}

We now replace the sampling efficiency rules $(\S 3.2)$ for modifying $1 \%$ database lightcurves with a new set of rules derived from the PRFs. The PRFs offer us an empirical set of rules that realistically incorporate blending and the many systematic photometry effects observed in SoDoPhot that were described in the last section.

To add microlensing onto a lightcurve with the PRFs we must first match a lightcurve to a PRF. This is most consistently performed using only the available measured parameters of the lightcurves, such as magnitude, color, average crowding, average error, etc. We have limited ourselves to three parameters, $V_{\text {obj }}, V-R_{\text {obj }}$, and crowding for the match. A match in average error was not chosen as it is already highly correlated with magnitude. Instead we chose to match in crowding for the following reasons, (1) the average error is also correlated with crowding (independent of magnitude), (2) the crowding parameter is a natural measure for parameterizing the image plane (i. e., regions of high stellar density versus low stellar density) as there is a strong correlation $(r=0.95)$ of the average crowding in MACHO fields with stellar density, and (3) of all the measured parameters, we believe crowding to be the most likely to be connected with blending. Though we see no strong correlations between blending and crowding, we do see a bifurcation reminiscent of Figure 9 with a slight preference for strongly blended events to also be highly crowded.

To match our two large databases (1\% database and PRDB database) as uniformly as possible we use the following scheme. First, the $1 \%$ database of lightcurves is sampled uniformly because of the importance of correctly weighting the temporal sampling and the unbiased nature of the database. For each lightcurve in the $1 \%$ database a robust mean magnitude $V_{\mathrm{obj}}$, color $V-R_{\mathrm{obj}}$, and crowding $C_{\mathrm{obj}}$ are computed. The PRFs are binned in three dimensions corresponding to their baseline magnitude $V_{P R F}$, color $V-R_{P R F}$, and crowding $C_{P R F}$, with bins sizes of $\sim 0.2$ magnitude $\left(N_{\text {bins }}=28\right), \sim 0.1$ color $\left(N_{\text {bins }}=17\right)$, and $\sim 30$ crowding $10\left(N_{\text {bins }}=\right.$ $7)$. The bin sizes are constant over the most dense regions of their respective distributions, but grow slightly in size near the edges to accommodate for sparseness. The mean number of PRFs in each bin is $\sim 17$. However, due to the shape of the CMD $\sim 51 \%$ of these bins are empty (as they should be) and thus the mean number of PRFs in occupied bins is $\sim 34$, with the typical occupied bin containing 12 20 PRFs. With the large number of PRFs employed it is unlikely $(\sim 1 \%)$ that a lightcurve will encounter an empty bin. When this does occur we simply match to the nearest non-empty bin (while holding crowding constant) which is never more than one bin away. The PRF bin that best matches the lightcurve is then selected and a PRF is randomly chosen from this bin. In this way we sample the PRFs as uniformly as possible.

Once a PRF has been matched to a lightcurve, event parameters $A_{\max }, t_{\max }$, and $\hat{t}$ are generated as in $\S 3.2$. The event is now added onto the lightcurve using the rules from the PRF. Each observation on the lightcurve with $A(t)>1.005$ is matched to the closest corresponding observing condition, $i$, in the PRF. In practice this amounts to minimizing the quantity $\Delta s=(\Delta \text { seeing })^{2}+(\Delta s k y)^{2}$ to determine $i$, where $\Delta$ seeing is the difference between the observation's seeing and the seeing in one of the $\sim 69$ observing conditions contained in the PRF, normalized to the maximum range of seeing. $\Delta s k y$ is similarly defined and normalized. ${ }^{10}$ The crowding parameter ranges from 0 to 255 and is logarith-
mic in nature. 
Having determined the observing conditions $i$, a two point linear interpolation is used to compute $A_{\text {rec }}$ based on the low point $A_{l o}=A_{r e c}(i, j)$ and high point $A_{h i}=A_{r e c}(i, j+1)$ that bracket $A(t)$ (or the lowest two points for an extrapolation). To preserve as much of the intrinsic lightcurve scatter as possible a technique similar to that outlined in $\S 3.2$ is employed in magnifying the flux. That is each magnitude is modified as $m \rightarrow \bar{m}-2.5 \log \left(A_{\text {rec }}(u(t))-1+10^{(\bar{m}-m) / 2.5}\right)$. The relative error bars are modified as $\sigma \rightarrow A_{\sigma} \sigma$, where $\sigma$ is the relative error bar for this observation and $A_{\sigma}$ is also computed via a linear interpolation between $A_{l o}$ and $A_{h i}$ from the tabulated values of $A_{\sigma}(i, j)$ ( $\left.\$ 4.3\right)$. This technique preserves the intrinsic size of the lightcurve's relative error bars and realistically modifies how they respond with $A$ over the various observing conditions.

The PRFs also allow us to modify the photometry flags recorded by SoDoPhot. As discussed in $\S 4.3$ some of these show clear signs of systematic behavior with magnification. We chose to modify (1) the crowding parameter, which represents the amount of flux contributed from nearby stars, (2) the $\chi_{P S F}^{2}$, which tends toward worse fits in better seeing and high magnification, and (3) the fit sky value. We do not modify (4) the object type flag, (5) the weighted fractions of the PSF masked due to cosmic rays, and (6) the weighted fractions of the PSF masked due to bad pixels.

The flags are modified as Flag $\rightarrow$ Flag - dFlag, where Flag is the respective flag (crowding, $\chi_{P S F}^{2}$, or fit sky) and dFlag is a linearly interpolated difference between the PRF's flag value at $A$ and at $A=1.0$, computed separately for each observing condition $i$. Because the flags are logarithmic in nature the magnification translates into an additive term, much like in the case of magnitudes. This technique preserves the lightcurve's intrinsic flag values while realistically altering them as a function of $A$ and observing conditions.

A sample lightcurve modified using the four PRFs displayed in Figures 7 and 8 is illustrated in Figure 10 . In each panel the input event parameters were the same, $A_{\max }=2.0$ and $\hat{t}=80.0$ days. The panels correspond to blend fractions (a) $m=1.0$, (b) $m=0.77$, (c) $m=0.65$, and (d) $m=0.35$. Note the dramatic drop in magnification $A_{\max }$ and relative shortening of the duration $\hat{t}$ as the event becomes more blended. Also note the chromatic behavior exhibited in PRF (c) due to the difference in color between the lensed star and the blended flux. We have visually inspected a large number of artificial lightcurves and compared the modified portions with real events seen towards both the LMC and Galactic Bulge. The comparison of magnification, error bars, and flags and their correlations with seeing and magnification are all quite satisfactory.

\section{RESULTS OF THE PHOTOMETRIC EF- FICIENCY}

A procedure similar to that outlined in $\S 3.3$ is used to compute the photometric efficiency, with one important difference. In $\S 3.3$ the distribution in luminosity of the stars was assumed to be the same as that observed by the survey (i.e., uncorrected for incompleteness) since all photometered objects were counted as resolved stars. Since we are now adding events onto lightcurves using an underlying luminosity distribution we need to integrate over this distribution, as the observed distribution is clearly incorrect $(\S 4.1)$. In principle, one could quantify the efficiency as a function of both duration $\hat{t}$ and luminosity $V$, and use this directly. However, in practice we cannot unambiguously determine the unlensed luminosity (at least without additional intensive follow-up photometry for blending fits or photometry from space). Since the LMC's LF is well known to $V \sim 24$ we opt to integrate out this variable.

To generate adequate statistics we make 10 passes through the $1 \%$ database of lightcurves, matching each lightcurve to a PRF, which inturn is used to modify the lightcurve $(\S 4.4$. Bookkeeping information (lightcurve id, PRF id, $V, V-R, V_{\mathrm{obj}}, V-R_{\mathrm{obj}}$, $A_{\max }, t_{\max }, \hat{t}$, etc.) for all lightcurves is stored and matched to the corresponding statistics $\left(A_{\text {max }}^{\prime}, t_{\text {max }}^{\prime}\right.$, $\hat{t}^{\prime}$, etc.) generated by the time-series analysis $(\S 2)$. Selection criteria set A and B are applied to these statistics and the results stored as integers in the datacube.

\subsection{Efficiency}

The datacube is binned in two dimensions, $V$ and $\hat{t}$. Recall that events are added uniformly in $t_{\max }$ and $u_{m i n}$, and so these are averaged over. We choose 100 bins in $V$ of size $0.1 \mathrm{mag}$ in the range $15<V<25$, and 24 bins in $\hat{t}$ that are logarithmically spaced in the range $1.0<\hat{t}<2000.0$ days (Table 3). Our artificial events are added uniformly in $\log (\hat{t})$, so bins logarithmically spaced insures equal numbers of events in 
each $\hat{t}$ bin. For each bin an efficiency is computed: $\epsilon(\hat{t}, V)=N_{\text {rec }}(\hat{t}, V) / N_{\text {add }}(\hat{t}, V)$ where $N_{\text {rec }}(\hat{t}, V)$ and $N_{\text {add }}(\hat{t}, V)$ are the number of events that pass the selection criteria set and the number of added events in the bin of $\hat{t}$ and $V$, respectively. It is worth mentioning that because $\epsilon(\hat{t}, V)$ is computed separately in each $V$ bin, this function is independent of the CMD used to seed the artificial star tests. From 10 passes through the $1 \%$ database, $\sim 1.2$ million artificial events are generated and of these only $\sim 43,000$ pass criteria set A and $\sim 52,000$ pass criteria set B. The mean number of added events per bin is 500 and the mean number of recovered events per bin is 18 (criteria set A).

The function $\epsilon(\hat{t}, V)$ for criteria set B is shown in Figure 11 as a contour plot in panel (b). The contours correspond to efficiencies of $0.001,0.01,0.1,0.2,0.3$, 0.4 , and 0.5 . The gross behavior with $\hat{t}$ and $V$ is apparent, with a broad peak in efficiency over the ranges $30<\hat{t}<200$ days and $17<V<19.5$. The function $\epsilon(\hat{t}, V)$ falls off rapidly for magnitudes smaller than $V \sim 17$ due to an explicit cut $V>17$ (criteria set $\mathrm{B})$, but the more gradual fall off for dim stars is a natural consequence of fainter, lower $\mathrm{S} / \mathrm{N}$ events. The gradual drop in efficiency for short duration events is caused by the sampling issues discussed in $\S$ 3.4. The sharp cut at long durations ( $\hat{t}<600$ days) seen in the sampling efficiency has here disappeared, although a remnant can still be seen for bright events. The cause of this, of course, is blending. Bright events are far less likely to be heavily blended, and thus are unlikely to be recovered with $\hat{t}$ 's longer than 600 days. Events that are fainter are more commonly blended (fit $\hat{t}$ 's much shorter) and can be scattered below the cut at 600 days. A corollary to this is that intrinsically short events are unlikely to be detected on faint stars, which are typically blended, as is also seen in Figure 11, panel (b).

Given the uncertain knowledge of the source star's luminosity, the somewhat noisy function $\epsilon(\hat{t}, V)$, and the fairly well constrained shape of the LMC's LF, we have opted to integrate out the variable $V$ in the function $\epsilon(\hat{t}, V)$. Although the shape of the LMC's $\mathrm{LF}$ is well known, the overall normalization to our fields, that is the number of stars with $V<24$ in our fields, is less certain $(\S 4.1)$. This uncertainty in normalization translates into an uncertainty in the survey's exposure in star-years. However, it is expected that the efficiency times the exposure will converge at some magnitude $V_{\text {stop }}$. Furthermore it is convenient to refer to our 'exposure' in units of objects-years (the number of lightcurves monitored times the duration of the survey) as this number is well known. We opt to move the (uncertain) normalization, that translates the 'exposure' in object-years into exposure in staryears, into the efficiency. We can understand this as follows. Assume a general distribution of event durations $d \Gamma / d \hat{t}$ for some Galactic model. The number of expected events $N_{\text {exp }}$ is just,

$$
N_{\text {exp }}=E_{*} \int_{0}^{\infty} \mathcal{E}_{*}(\hat{t}) \frac{d \Gamma}{d \hat{t}} d \hat{t},
$$

where the exposure here, $E_{*}$, is in star-years and the efficiency is calculated from $\epsilon(\hat{t}, V)$ as,

$$
\mathcal{E}_{*}(\hat{t})=\int^{V_{\text {stop }}} \epsilon(\hat{t}, V) \phi(V) d V .
$$

Here $V_{\text {stop }}$ is some cut-off magnitude where the integration is stopped and $\phi(V)$ is the LMC's LF normalized such that,

$$
\int^{V_{\text {stop }}} \phi(V) d V=1.0
$$

The exposure, $E_{*}$, in Equation 6 must be related to the observed number of objects, $N_{\text {obj }}$, as,

$$
E_{*}=\left[S / O\left(V_{\text {stop }}\right)\right] N_{\mathrm{obj}} T,
$$

where $T$ is the time-span of the survey and $S / O\left(V_{\text {stop }}\right)$ is a scaling factor that converts the average number of objects seen in the survey to the actual number of stars, down to the cut-off magnitude $V_{\text {stop }}$. $S / O\left(V_{\text {stop }}\right)$ may be estimated from,

$$
S / O\left(V_{\text {stop }}\right)=\frac{\int^{V_{\text {stop }}} \Phi(V) d V}{\int^{V_{\text {stop }}} \Phi_{\text {obj }}\left(V_{\text {obj }}\right) d V_{\text {obj }}},
$$

where $\Phi_{\mathrm{obj}}\left(V_{\mathrm{obj}}\right)$ is the ground-based luminosity function and is normalized to the total number of objects observed by the survey: i.e., $\Phi_{\text {obj }}\left(V_{\text {obj }}\right)=$ $N_{\text {obj }} \phi_{\text {obj }}\left(V_{\text {obj }}\right)$. Similarly $\Phi(V)$ is the true underlying LF normalized as in $\S$ 4.1.2. The normalization $S / O\left(V_{\text {stop }}\right)$ was estimated for each field in $\S 4.1$ (Table 1 and Figure 5 ) for two cut-off magnitudes $V_{\text {stop }}=22$ and $V_{\text {stop }}=24$ and for two LF $\left(L F_{1}\right.$ and $\left.L F_{2}\right)$. We make a simple re-definition; let $\mathcal{E}(\hat{t})=\left[S / O\left(V_{\text {stop }}\right)\right] \mathcal{E}_{*}(\hat{t})$ and $E=N_{\text {obj }} T$. With these definitions Equation 6 can be written as, 


$$
N_{\exp }=E \int_{0}^{\infty} \mathcal{E}(\hat{t}) \frac{d \Gamma}{d \hat{t}} d \hat{t} .
$$

A similar re-definition may be performed on Equation 11 for the optical depth. The advantage of using $E$ and $\mathcal{E}(\hat{t})$ instead of $E_{*}$ and $\mathcal{E}_{*}(\hat{t})$ is twofold. Firstly, the exposure in object-years, E, is known accurately. Secondly, this substitution leaves $\mathcal{E}(\hat{t})$ containing the only reference to $V_{\text {stop }}$ (implicitly) and we can investigate its convergence with magnitude easily. It is important to note that with the above definition of $\mathcal{E}(\hat{t})$, the efficiency is no longer bound to lie below one. This slightly un-intuitive result is due to the fact that $S / O\left(V_{\text {stop }}\right)$ may be quite large (Table 1), though in practice $\mathcal{E}(\hat{t})$ always lies below one. A practical way of viewing $\mathcal{E}(\hat{t})$ is: given $S / O\left(V_{\text {stop }}\right)$ events, $\mathcal{E}(\hat{t})$ is the expected number of detected events for the given $\hat{t}$. In the limit $S / O\left(V_{\text {stop }}\right) \rightarrow 1.0$ the efficiency $\mathcal{E}(\hat{t})$ recovers its usual meaning.

Figure 11 illustrates the integration of $\epsilon(\hat{t}, V)$, panel (b), over a LF, panel (a). The resulting function $\mathcal{E}(\hat{t})=[S / O(V<24)] * \mathcal{E}_{*}(\hat{t})$ is shown in panel (c). Our two LFs, $L F_{1}$ and $L F_{2}$, and their corresponding values of $S / O(V<24)$ are displayed as dotted and solid lines, respectively. An important result is that $\mathcal{E}(\hat{t})$ is fairly robust to uncertainties in the LF fainter than $V \sim 22$. For durations less than 75 days the difference in $\mathcal{E}(\hat{t})$ as derived using either LF is less than $1 \%$. The difference, however, becomes progressively larger for longer durations due to the relative difference in contributions from faint stars exhibited by the two LFs. At 300 days the difference is $\sim 3 \%$. In $\S 4.1$ we chose to favor $L F_{2}$ over $L F_{1}$ because of our HST completeness tests and because it seems unlikely that the LF will continue to rise so steeply beyond the clump for so long. Evidently any moderately different LF would produce only a small difference in the overall efficiency.

In Figure 12 we present the efficiency $\mathcal{E}(\hat{t})$ for selection criteria set $\mathrm{A}$ and $\mathrm{B}$ using $\mathrm{LF} L F_{2}$. Also shown for comparison are the photometric efficiencies used in A96 and A97. Many of the differences between the old and new results, as seen in the sampling efficiencies $(\S 3.4$ ), can also be seen here. Again the most striking difference is the much larger efficiency at long durations. As described in $\S 3.4$ this has multiple causes, including (1) almost three times more baseline in the A00 data than in the A97 data, (2) a longer duration cut of $\hat{t}<600$ days and (3) 6 high density fields which were previously split into two sep- arate years of data (due to an early generation of templates) have been recombined. A new reason, unique to the photometric efficiency, is the contribution of faint stars to the efficiency for long duration events. This effect was troublesome in the A96 and A97 results since we lacked adequate numbers of faint stars (only a few with $V \sim 22$ and none with $V>22$ ) and thus were only confident that the photometric efficiency had converged for durations less than 150 days. As we show below the A97 results were likely to have converged only for durations less than 100 days. However, this resulted in an underestimate in the efficiency of less than $10 \%$ for durations around 150 days. Criteria set A is closer in design to the A97 cuts and, as a consequence, follows the older results more closely for very short durations up to about 60 days. As noted above the sharp cut-off at durations around 600 days seen in the sampling efficiency is smoothed over in the photometric efficiency because blending scatters intrinsically long duration events to shorter measured durations.

\subsection{Convergence}

Figure 13 shows the convergence of $\mathcal{E}(\hat{t})$ with $V_{\text {stop }}$ for the four combinations of selection criteria sets $A$ and $\mathrm{B}$ and $L F_{1}$ and $L F_{2}$. In each panel the convergence of four different durations $(\hat{t}=50,100,300$, and 1000 days) is shown. The ordinate is in arbitrary relative units. Criteria set B is our loosest set of cuts and is the least convergent of the two selection criteria sets used in A00. An inspection of the figure also shows that $L F_{1}$ gives a somewhat slower convergence than $L F_{2}$. This is not surprising as $L F_{1}$ contributes a substantially larger number of fainter stars as compared with $L F_{2}$. Criteria set A with $L F_{2}$ converges for durations less than 1000 days by $V_{\text {stop }} \sim 24.5$ and criteria set $\mathrm{B}$ with $L F_{2}$ converges for durations less than 300 days by $V_{\text {stop }} \sim 24$. If the true LF is closer in form to $L F_{1}$ then it is possible that our efficiency results are somewhat underestimated for long duration events (see $\S 5.3$ below). However, since none of the 17 candidates in A00 have durations longer than 300 days we feel that the convergence of the efficiency is more than adequate.

\subsection{Error Budget}

There are a number of potential sources of error in estimating our efficiency and we list the most important ones in Table 1 . We crudely classify the errors as 'signed' or 'unsigned'. That is, if we are reasonably 
certain the effect would only increase (or decrease) the efficiency we classified it as 'signed' and marked it with the appropriate sign in the table. If we are not certain of it's sign we called it 'unsigned' and left a question mark in the sign column of the table.

The first unsigned error in the table (\#1) is simply the uncertainty in our normalizations and was estimated to be $\sim 20 \%$ in $\S 4.1 .2$. That is we are confident that we know the number of stars to the limit $V=24$ in our LMC fields to within $\sim 20 \%$. This is by far our largest source of error in the efficiency. The second unsigned error (\#2) is an uncertainly in our incomplete knowledge of the shape of the LMC's LF at faint magnitudes. In $\S 5.1$ we used the difference between $L F_{1}$ and $L F_{2}$ to estimate a $1-3 \%$ effect depending on the duration of the event. Another source of unsigned error $(\# 3)$ is due to the finite number of Monte Carlo events, which we estimate to be $\lesssim 1 \%$ based on the binomial distribution and the number of Monte Carlo events used.

The fourth entry in Table $(\# 4)$ is signed and represents a potential over-completeness bias. Because blending might over-populate objects with respect to stars in our calibration bin $(17.5<V<18.5)$, this could cause an overestimation of $\mathcal{E}(\hat{t})$. In $\S 4.1 .2$ we estimated this could be as large as $\sim 9 \%$, but likely to be somewhat smaller (perhaps 6-8\%). The next signed error (\#5) is related to our choice of using only the nearest neighbor to each artificial star, when in fact as many as $~ 3 \%$ of PRFs had 2nd or 3rd nearest neighbors which were more sensitive to the lensed flux. Of course what matters is how many 2nd or 3rd nearest neighbors would have been detected but were not because the closest neighbor was used instead, and this is probably smaller than the number of 2 nd and 3rd nearest neighbors who are more sensitive. The efficiency is likely to be underestimated somewhat by this error. The next two signed errors are small and relate to (\#6) our use of a 'universal' LF (especially in the outer LMC disk fields where the true LF likely turns over at brighter magnitudes) and $(\# 7)$ a possible slight underestimation of $\mathcal{E}(\hat{t})$ if convergence has not been reached by $V=24$ (e. g., if the the true underlying luminosity function is closer to $L F_{1} ; \S 5.2$.

We leave two potential sources of error unexplored in this paper because of their highly uncertain and complicated nature. The first of these (\#8 in the table) is the difficulty of incorporating binary star systems into the determination of the LF. Binary stars could increase our exposure (thereby increasing $\mathcal{E}(\hat{t})$ ) but also shift the LF toward fainter magnitudes (decreasing $\mathcal{E}(\hat{t}))$. The effect on the LF of the local neighborhood is still controversial (Kroupa 1995, Reid \& Gizis 1997) and any hope of resolving this for the LMC is beyond the scope of this paper, and left for future work. Another unexplored source of error (\#9) is due to our lack of exotic lensing Monte Carlo events. The behavior of this source of error is difficult to estimate as the shape of exotic lensing is likely to lower our efficiency, but caustic crossings might well increase it (Di Stefano \& Perna 1997, Di Stefano 1999). Binary lensing events are likely to dominate this effect, but the distributions of their event parameters is wide open to speculation (but see Alcock et al. 1999c). We plan to investigate the effects of a binary lens population on our efficiency in a future paper.

Given the generally small size of the individual signed errors relative to the size of the unsigned errors, as well as the Poisson-like 'counting' errors in the optical depth estimates (13-17 events gives $\sim 30-40 \%$ errors in the optical depth; see Table 10 in A00), halo mass fraction, etc., and the fact that a number of these signed errors are of comparable size but opposite sign, we have chosen not to attempt to correct for them. Our estimates of their size are rough, but probably accurate to within a factor of two. There is no correct way to total systematic errors - but assuming they are uncorrelated we can sum them in quadrature to get a total of $\sim 22 \%$, not including errors \#8 and \#9, which may be the largest, but for which we do not have good estimates. Alternatively, we can add the signed errors algebraically to get $\sim 5 \%$, then to this add the unsigned errors in quadrature, giving $\sim 21 \%$ total error. So our best guess at the size of our error is $21-22 \%$ plus the unknown effects of binary corrections to the LF and exotic microlensing.

\subsection{Efficiency Differences Across Fields}

Our efficiency for each field is shown in Figure 14. These have been calculated in the manner described above and normalized with each field's $S / O$ (Table 1). By splitting our $~ 1.2$ million Monte Carlo events into 30 fields, the shot noise has increased to $5-10 \%$, which is somewhat field dependent. In principle the shot noise can be reduced by simply running more passes, but the CPU time required is somewhat prohibitive at the present time. Nevertheless the efficiency for each field is important when looking for gradients in opti- 
cal depth or rates across the face of the LMC, which the present level of accuracy is adequate for, given the small number of events (13-17) seen toward the LMC. Differences seen between fields in Figure 14 are due primarily to (1) different normalizations for each field (Table 1), physically corresponding to larger numbers of stars and thus probability of detecting events in some fields, (2) different sampling rates due to observing strategy and weather across fields, and (3) to a smaller degree more crowded fields tend to have a larger effective area (area covered by stellar PSFs in the image plane) in which to detect the presence of magnified events, because of blending.

\subsection{Parameter Bias}

As discussed in the introduction, blending induces a bias in the measured parameters $A_{\max }$ and $\hat{t}$. We can quantify this bias using our Monte Carlo events, and have developed a method of statistically correcting for $\hat{t}$ bias when calculating optical depths. Figure 15 plots the ratio $A_{\max }^{\prime} / A_{\max }$ versus $\hat{t}^{\prime} / \hat{t}$ for a random sample $\left(N \sim 10^{5}\right)$ of Monte Carlo events: parlel (a) is for sampling efficiency events and (b) is for photometric efficiency events (both use selection criteria set B). The majority of sampling efficiency events are recovered with little or no parameter bias in L (There is a small amount of bias in A both directions) caused by sampling and weather gaps that effectively obfuscate very high magnification events.) As the sampling efficiency does not simulate blending this result is not surprising. The results for the photometric efficiency events is quite different. A significant amount of blending can be seen in panel (b) of Figure 15, were approximately $40 \%$ of the Monte Carlo events are blended with an additional $10 \%$ or more of un-lensed flux. The trend of decreasing $A_{\max }$ with decreasing $\hat{t}$ for highly blended events is well delineated in the figure. Note that the photometric efficiency events sample the CMD (luminosity function) in a square-root fashion ( $\S 4.2$ ) and thus dim stars are underpopulated in panel (b) of Figure 15. Factoring this in, as is done below, further increases the amount of blending and the size of any potential correction.

We can calculate the efficiency as a function of various parameters (e.g., $u_{m i n}^{\prime}, u_{m i n}, V, V_{\text {obj }}$, etc.) for the photometric efficiency, and some of these are shown in A00 (for example, see their Figure 6). However, since the Monte Carlo events sample the CMD in a square-root fashion we must bin and re-weight with the correct LF. We must also bin and re-weight with a realistic distribution in $\hat{t}$ as the Monte Carlo events are added with durations uniform in $\log (\hat{t})$, which is unlikely to be the true distribution. We chose to reweight with the distribution of durations predicted by a standard halo model with delta function mass $0.5 M_{\odot}$ (Griest 1991), since this distribution closely matches the data (average duration $\hat{t} \sim 92$ days; see A00). Fortunately, model dependency is weak as the distributions in $u_{\min }$, weighted and un-weighted (that is using the $\log (\hat{t})$ distribution) are quite similar.

If the $\hat{t}$ bias is left uncorrected the optical depth will be underestimated (Equation 11). There are two very different, but complimentary, ways to correct for this bias in $\tau_{\text {meas }}$. Ideally one could perform microlensing fits on individual events that allow for unlensed flux in each passband. Then each event could be corrected for blending separately and the maximum amount of information in the set of $\hat{t}$ 's would be retained. In practice this approach is difficult as the lightcurves are not always well sampled and can have considerable photometric scatter making the blend fits uncertain (Alcock et al. 1996, Pratt 1997, Wozniak 1997, Han 1998). This situation can be greatly improved with accurate, dense, follow-up photometry on alerted events as is being done now routinely (Albrow et al. 1998a, Alcock et al. 19979, Rhie et al. 1999). However, not all events are alerted. In addition, blending fits are not always unique and only on fairly high magnification events can the fit parameters be extracted with confidence. A second approach is to correct the $\hat{t}$ bias in a statistical fashion. Since we have a priori knowledge of the distribution of source stars from the luminosity function $\phi(V)$ and can estimate the distribution of uncorrected durations $d \Gamma / d \hat{t}(\hat{t})$ we can compute a first order average correction as,

$$
\bar{\alpha}=\frac{\int d V \int d \hat{t} \alpha(\hat{t}, V) \epsilon(\hat{t}, V) \frac{d \Gamma}{d \hat{t}}(\hat{t}) \phi(V)}{\int d V \int d \hat{t} \epsilon(\hat{t}, V) \frac{d \Gamma}{d \hat{t}}(\hat{t}) \phi(V)},
$$

where $\epsilon(\hat{t}, V)$ is defined as above and $\alpha(\hat{t}, V)$ is defined as: $\alpha(\hat{t}, V)=\operatorname{median}\left(\hat{t}^{\prime} / \hat{t}\right)$ or the median $\hat{t}$ bias for the Monte Carlo events in a bin of $\hat{t}$ and $V$. The quantity $\alpha(\hat{t}, V)$ lies between zero and one and is a strong function of $\hat{t}$ and $V$ in the sense that $\alpha(\hat{t}, V)$ is one for bright events and approaches zero for both dim events and for long duration events. The results of Equation 12 for criteria set $\mathrm{A}$ and B using LFs $L F_{1}$ and $L F_{2}$ are tabulated in Table 0 , assuming mean 
durations of 41, 92, and 130 days (corresponding to delta-function masses of $0.1,0.5$, and $1.0 M_{\odot}$ in the model $d \Gamma / d \hat{t}(\hat{t})$, respectively). The mean duration of the microlensing candidates in A00 corresponds most closely with our value of $\hat{t} \sim 92$ days or an average mass of $M_{\odot} \sim 0.5$. This correction may be used in Equation 10 by simply substituting $\hat{t}^{\prime} \rightarrow \hat{t}^{\prime} / \bar{\alpha}$ to obtain,

$$
\tau_{\text {meas }} \rightarrow \frac{\pi}{4} \frac{1}{E} \sum_{i} \frac{\hat{t}_{i}^{\prime} / \bar{\alpha}}{\mathcal{E}\left(\hat{t}_{i}^{\prime} / \bar{\alpha}\right)} .
$$

Note the optical depth defined in this manner does not scale simply as $1 / \bar{\alpha}$. We have tried computing $\bar{\alpha}$ as a function of measured parameters (such as $V_{\mathrm{obj}}$ and $\hat{t}^{\prime}$ ) but find that it is fairly constant over the measured ranges. The use of a model distribution in $\hat{t}$ is somewhat worrisome. However, the range in corrections $\bar{\alpha}$ for different $\hat{t}$ distributions is acceptably small, about 5\% (Table 5). The error induced in the optical depth is somewhat smaller $(\sim 3 \%$; see Table 9 in $\mathrm{A} 00)$.

To check this statistical correction we ran a set of Monte Carlos on a toy model using our artificial events. The Monte Carlos work as follows: (1) first we bin the recovered artificial events in $\hat{t}$ and $V$, (2) we create $N_{\text {exp }}$ 'events' by sampling the $\mathrm{LF} \phi(V)$ and $\hat{t}$ distribution function $d \Gamma / d \hat{t}$ for a simple halo model with a delta function mass $0.5 M_{\odot}$ (model $\mathrm{S}$ in A96, $\mathrm{A} 97$, and A00), (3) for each of these $N_{\text {exp }}$ 'events' we chose an artificial event to represent it by selecting at random from the appropriate bin in $\hat{t}$ and $V$, (4) three different optical depths for these $N_{\text {exp }}$ 'events' are computed using three different $\hat{t}$ 's (the true $\hat{t}$, the fit $\hat{t}^{\prime}$ and the statistically corrected $\left.\hat{t}_{s t}=\hat{t}^{\prime} / \bar{\alpha}\right)$. The number of $N_{\text {exp }}$ 'events' corresponds to the predicted number of observed events as computed by Equation 2. The experiment is repeated 1000 times and the results for selection criteria set $\mathrm{B}$ and $L F_{2}$ are displayed as histograms of $\tau_{\text {meas }}$ in Figure 16. The dashed histogram corresponds to calculating $\tau_{\text {meas }}$ using the fit $\hat{t}^{\prime}$ 's and clearly underestimates the optical depth $\left(\tau_{\text {model }}=4.7 \times 10^{-7}\right.$ for Model S; the vertical line in the figure). Using the true durations $\hat{t}$ to compute $\tau_{\text {meas }}$ gives the correct optical depth (solid histogram). The optical depth computed using the statistically corrected durations, $\hat{t}^{\prime} / \bar{\alpha}$, is displayed as the dotted histogram and correctly predicts the true optical depth in a fairly unbiased manner. Similar unbiased results are found using selection cri- teria set $\mathrm{A}, L F_{1}, L F_{2}$, and using the $d \Gamma / d \hat{t}$ distributions with delta-function masses of 0.1 and $1.0 M_{\odot}$. These Monte Carlos give us confidence that this statistical correction can be used in an unbiased manner to compute the optical depth toward the LMC.

\section{SUMMARY}

The results of A00 for 5.7 years of photometric data toward the LMC rely critically on how well we understand the detection efficiency. In this paper we calculated these efficiencies, correcting the most important systematics effects with realistic models. To account for the wide range of issues due to blending we perform artificial star tests on a broad range of images. These artificial stars tests allowed us to empirically account for blending and to model our photometry code's systematics. Correcting for blending also required an accurate knowledge of the LMC's LF because microlensing magnifies flux and increases the survey's sensitivity to dim, unresolved stars.

We found that our sensitivity $\mathcal{E}(\hat{t})$ to dim, unresolved stars vanishes for magnitudes fainter than $V \sim 24$ and durations less than $\hat{t} \sim 300$ days. Our sensitivity in previous results (A96 and A97) was somewhat underestimated for durations greater than $\hat{t} \sim 100$ days. We also found the $\hat{t}$ bias, due to blending, is of the order of $20 \%$ and we presented a method for statistically correcting this bias in our optical depth estimates. The method is complimentary to using blended $\hat{t}$ fits.

\section{ACKNOWLEDGEMENTS}

We are very grateful for the skilled support given our project by the technical staffs at the Mt. Stromlo and CTIO Observatories, and in particular we would like to thank S. Chan, G. Thorpe, S. Sabine, J. Smillie, and M. McDonald, for their invaluable assistance in obtaining the data. We especially thank J.D. Reynolds for valuable assistance with the database software that has made this effort possible.

This work was performed under the auspices of the U.S. Department of Energy by University of California Lawrence Livermore National Laboratory under contract No. W-7405-Eng-48. Work performed by the Center for Particle Astrophysics personnel is supported in part by the Office of Science and Technology Centers of NSF under cooperative agreement AST8809616. Work performed at MSSSO is supported by the Bilateral Science and Technology Program of 
the Australian Department of Industry, Technology and Regional Development. DM is also supported by Fondecyt 1990440. CWS thanks the Packard Foundation for their generous support. WJS is supported by a PPARC Advanced Fellowship. KG is supported in part by the DOE under grant DEF03-90-ER 40546. TV was supported in part by an IGPP grant. 


\section{REFERENCES}

Alfonso, C. et al. 1998, A\&A, 337, L17

Alfonso, C. et al. 1999, A\&A, 344, L63

Albrow, M.D. et al. 1998a, ApJ, 509, 687

Albrow, M.D. et al. 1998b, ApJ, 512, 672

Alcock, C. et al. 1995, ApJ, 449, 28

Alcock, C. et al. 1996, ApJ, 461, 84

Alcock, C. et al. 1997a, ApJ, 486, 697

Alcock, C. et al. 1997b, ApJ, 491, L11

Alcock, C. et al. 1997c, ApJ, 491, 436

Alcock, C. et al. 1999a, ApJ, 518, 44

Alcock, C. et al. 1999b, PASP, 111, 1539

Alcock, C. et al. 1999c, ApJ, submitted astro-ph/9907369

Alcock, C. et al. 1999d, AJ, submitted astro-ph/0001435

Alcock, C. et al. 1999e, ApJ, submitted astro-ph/0001272

Ansari, R. 1997, A\&A, 324, 843

Crotts A.P.S., Uglesich, R., Gyuk, G., Tomaney, A.B., 1999, Astronomical Society of the Pacific Conference Series, 1999, 182, 409

Di Stefano, R. and Perna, R., 1997 ApJ, 488, 55

Di Stefano, R., 1999, ApJ, submitted astrop-ph/9901035

Geha, M. et al. 1998, ApJ, 115, 1045

Griest, K., 1991, ApJ, 366, 412

Griest, K. and Hu, W., 1992, ApJ, 397, 362

Groth, E.J. 1986, ApJ, 91, 1244

Han, C. 1997, ApJ, 490, 51

Han, C. 1998, ApJ, 500, 569

Kroupa, P., 1995, ApJ, 453, 358

Lasserre, T. et al. 2000, A\&A Letters, in press astro-ph/0002253
Mao, S. and Paczyński, B., 1991, ApJ, 374, L37

Marshall, S.L. et al. 1994, in IAU Symp. 161, 67

Olsen, K.A.G., Hodge, P.W., Mateo, M., Olszewski, E.W., Schommer, R.A., Suntzeff, N.B., Walker, A.R., et al. 1998, MNRAS, 300, 665

Olsen, K.A.G. 1999, ApJ, 117, 2244

Palanque-Delabrouille, N. et al. 1999, A\&A, 332, 1

Reid,N.I. and Gizis, J.E., 1997, AJ, 113, 2246

Rhie, S.H., Becker, A.C., Bennett, D.P., Fragile, P.C., Johnson, B.R., King, L.J., Peterson, B.A., Quinn, J., et al. 1999, ApJ, 522, 1037

Pratt, M. 1997, PhD Thesis, University of California at Santa Barbara \& University of Washington

Roulet, E. and Mollerach, S. 1997, Physics Reports, 279,68

Schechter, P.L., Mateo, M., and Saha, A. 1993, PASP, 105,1342

Stubbs, C.W. et al. 1993, Proc. SPIE, 1900, 192

Stetson, P.B. 1992, ASP Conf. Ser. 25, 297

Udalski, A. et al. 1997, Acta Astronomica, 47, 319

Wozniak, P. and Paczyński, B. 1997, ApJ, 487, 55

This 2-column preprint was prepared with the AAS LATEX macros $\mathrm{v} 4.0$. 


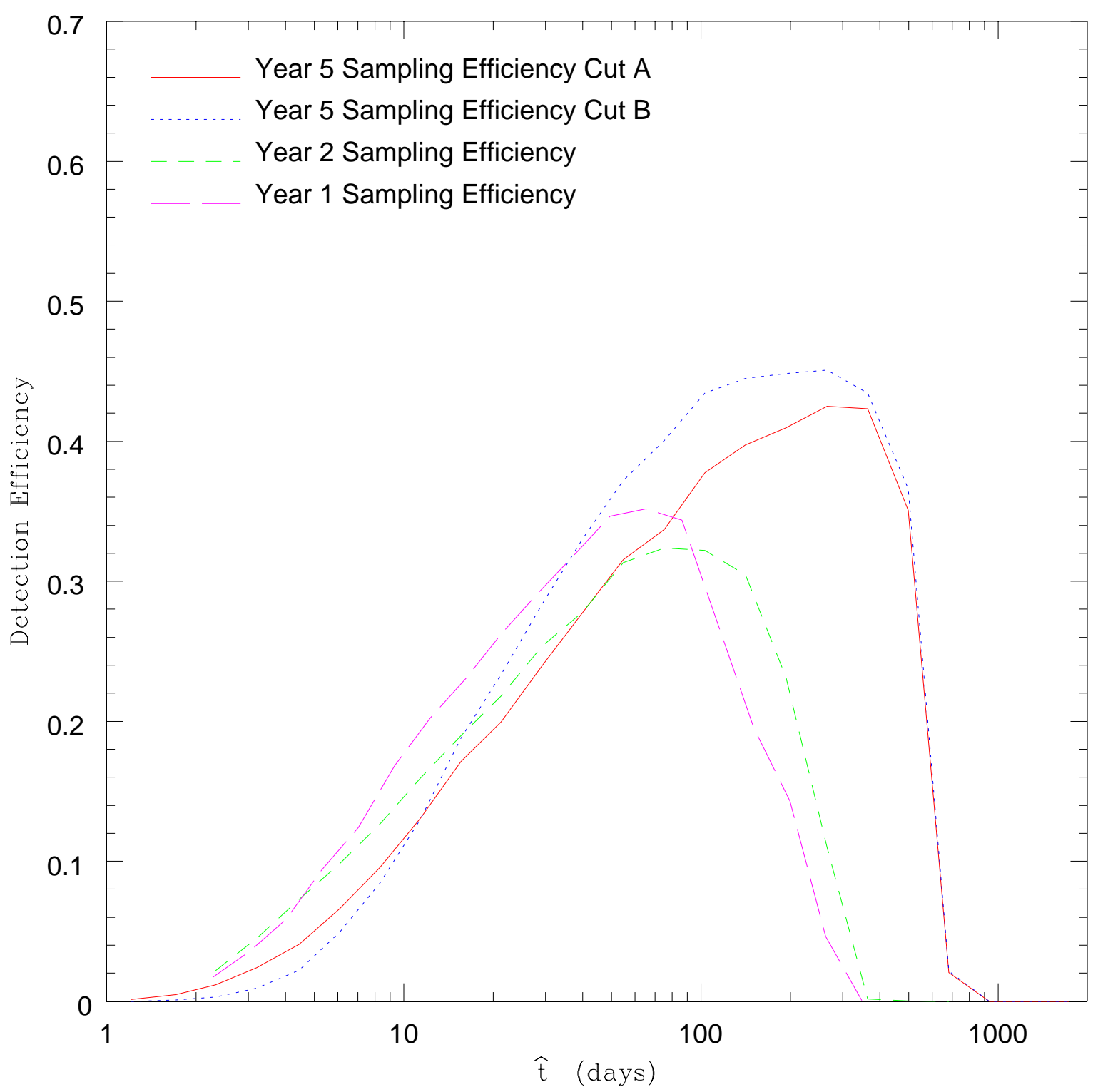

Fig. 1. - The 5.7-year sampling efficiency (normalized to $u_{\min }<1.0$ ) results for criteria set $\mathrm{A}$ (solid line) and criteria set B (dotted line). Also shown are the sampling efficiencies from A96 (long dash) and A97 (short dash) for comparison. 


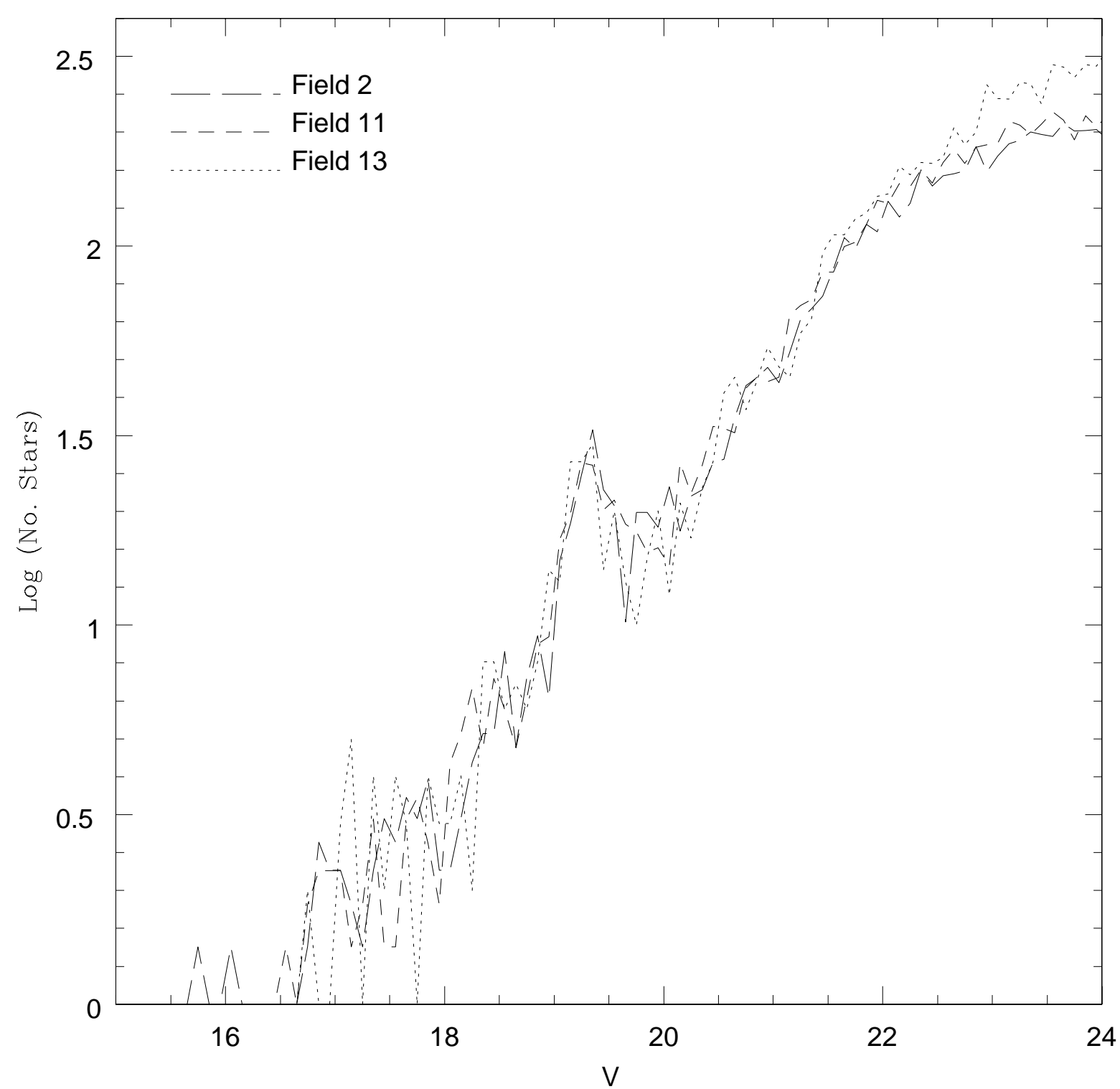

Fig. 2.- Three HST LFs of MACHO fields are plotted with line codings displayed in the figure. Details of the relative normalizations can be found in the text. Note the good match in shape from $V \sim 17$ to $V \sim 22$. 


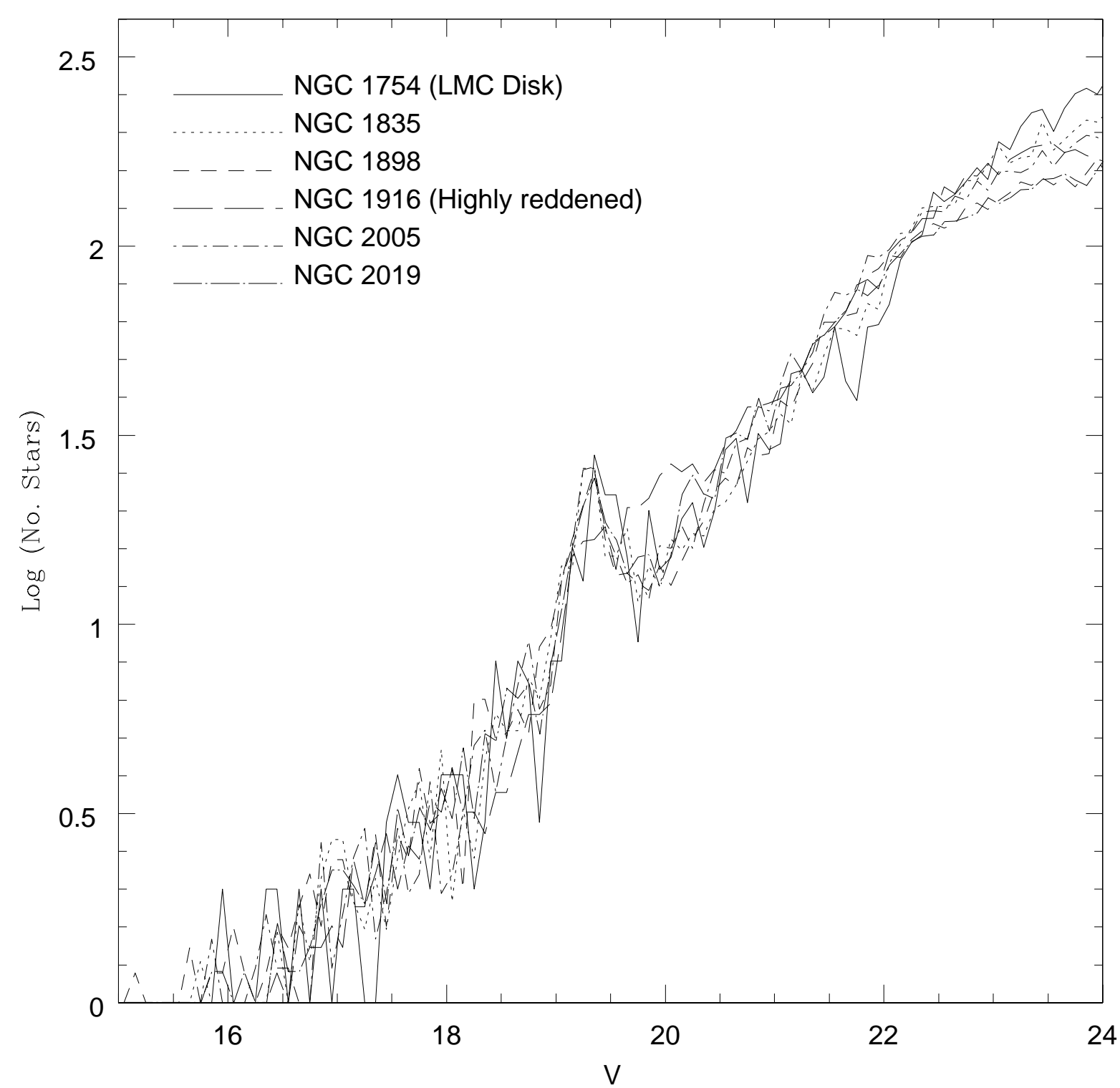

Fig. 3.- Six Olsen (1999) HST LFs are plotted with line codings as displayed in the figure. Details of the relative normalizations can be found in the text. Note the good match in shape from $V \sim 17$ to $V \sim 22$. 


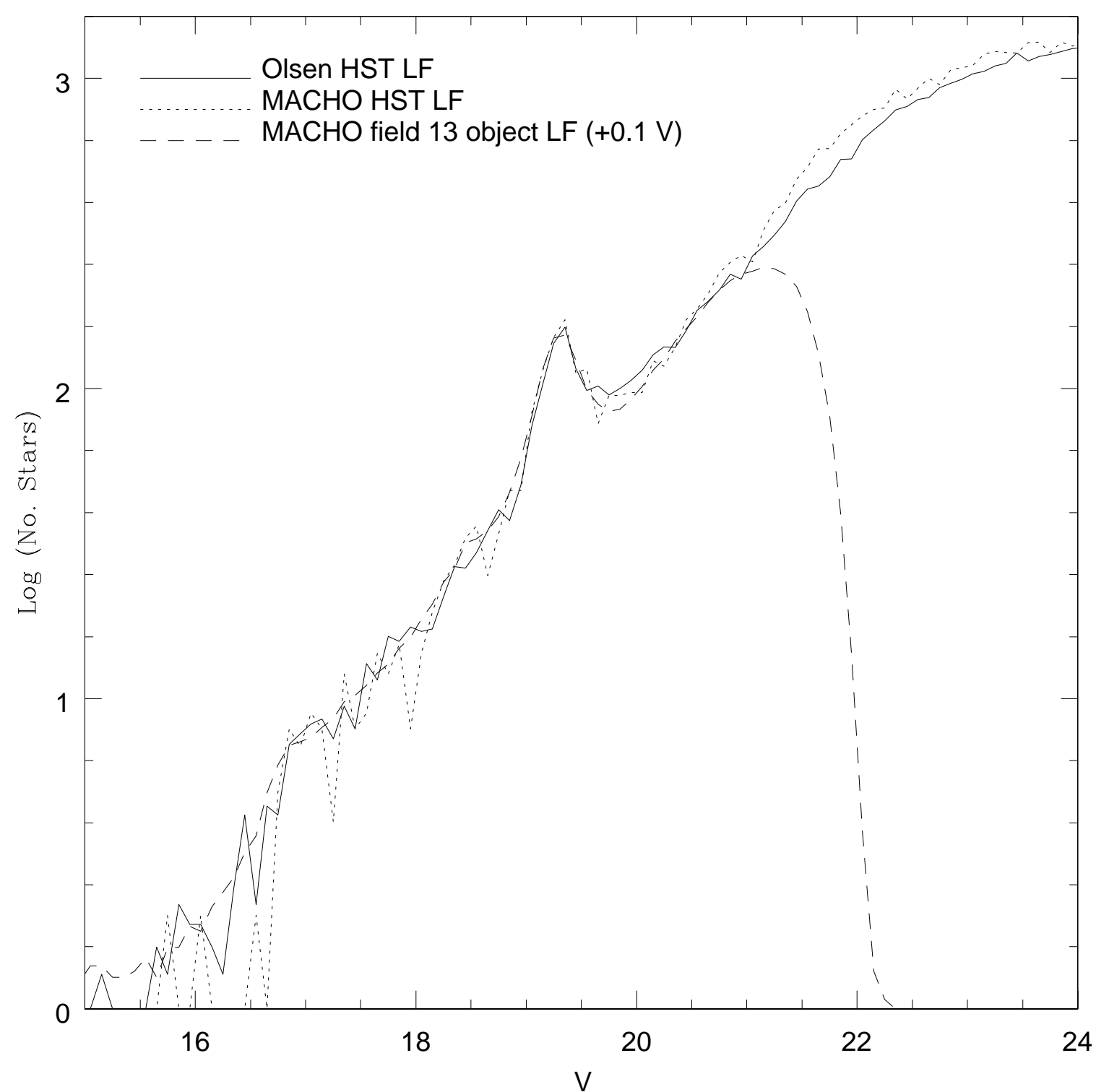

Fig. 4.- Combined MACHO HST LF (dotted line) is plotted and compared with the combined Olsen (1999) HST LF (solid line). Also shown is the ground-based LF for MACHO field 13 (dashed line) which have been normalized as described in the text. The agreement in shape over the range $17<V<21$ is good. 

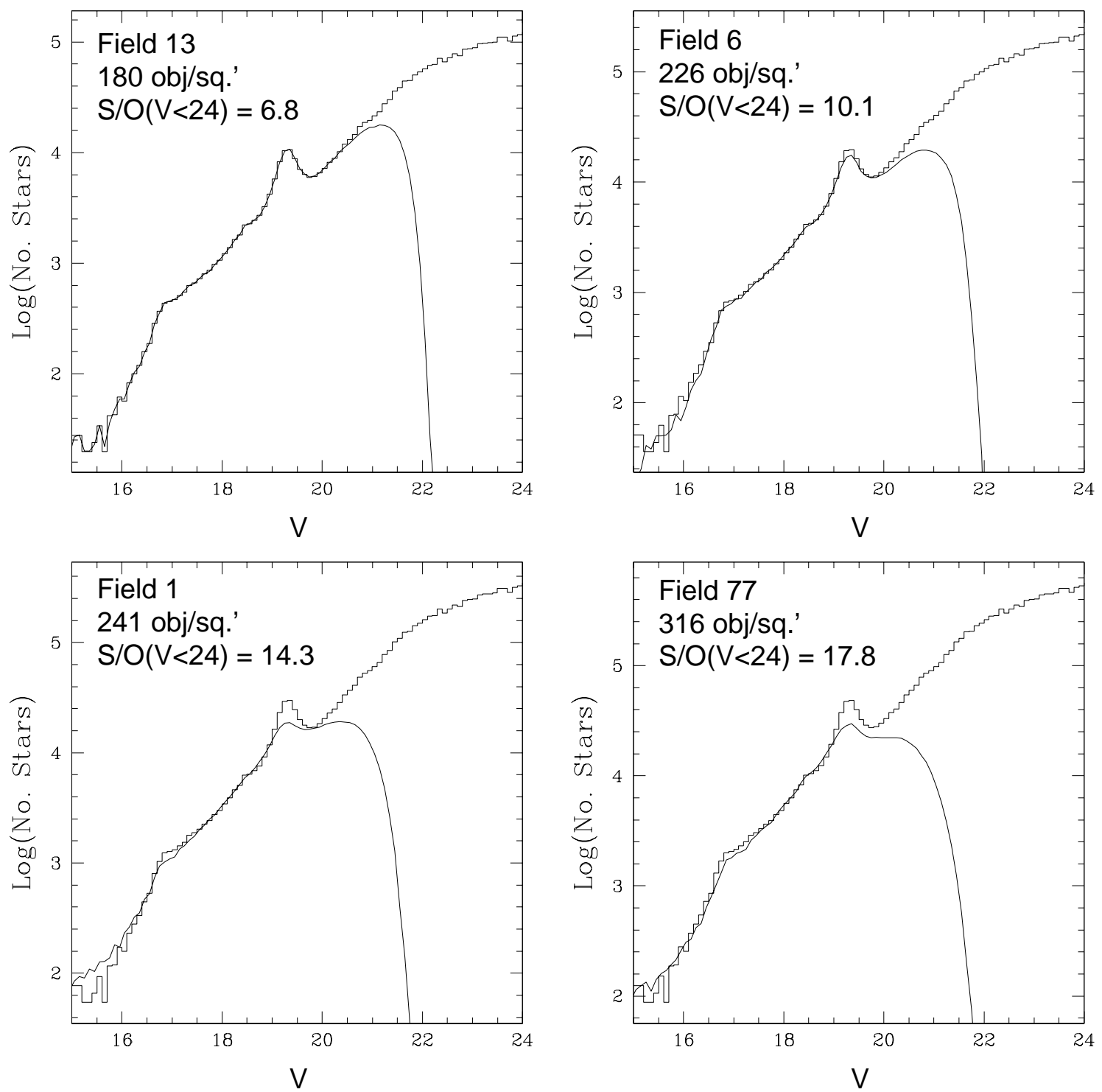

Fig. 5.- Four sample MACHO ground-based LFs (solid lines) ranging from low to high stellar density are labeled in the figure. $L F_{2}$ (histograms) has been offset and normalized (Table 1) to each field. The agreement in shapes between the individual ground-based LFs and $L F_{2}$ is quite reasonable. 


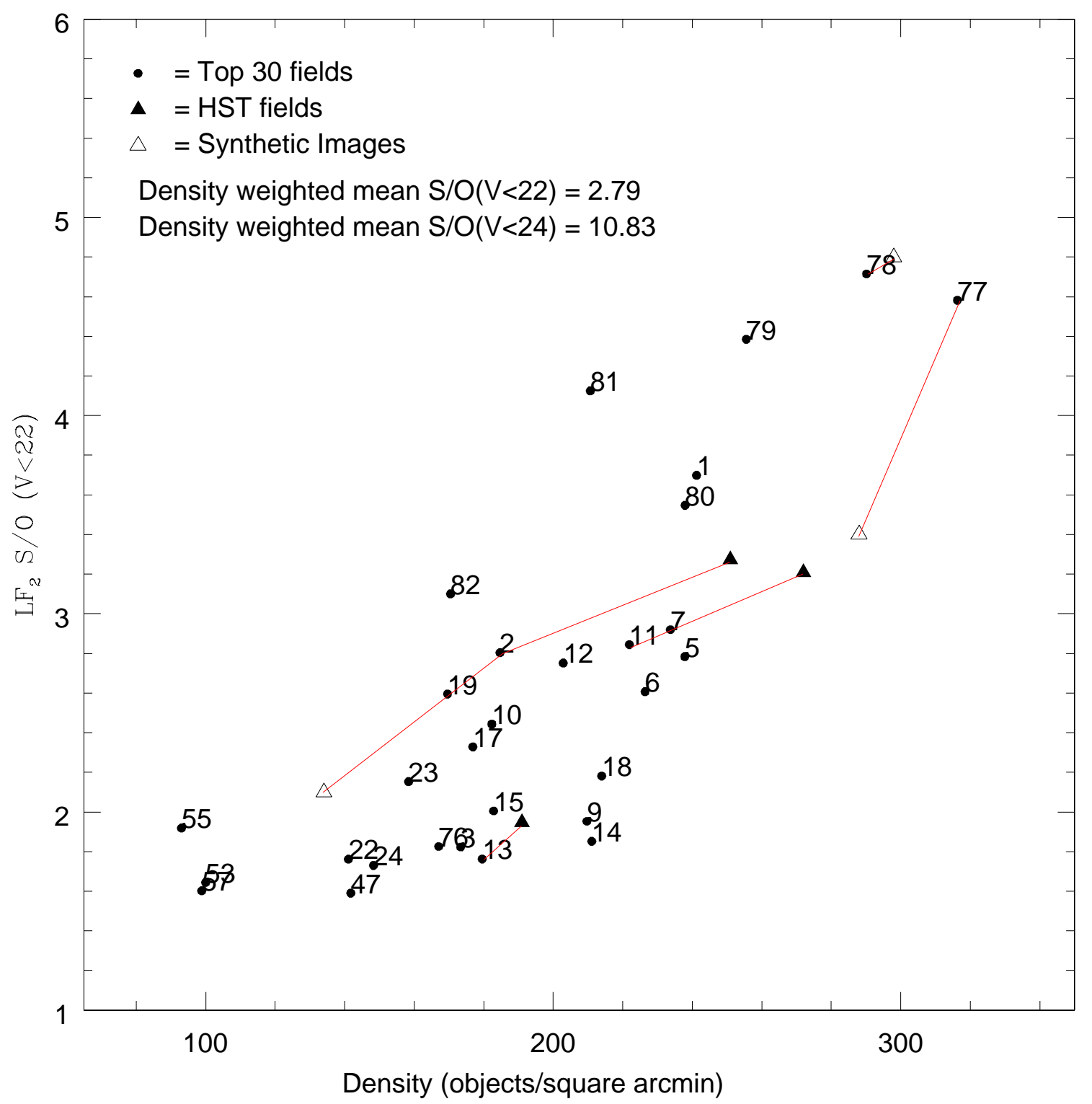

Fig. 6.- Normalization of each field as a function of object density. See text for details. 

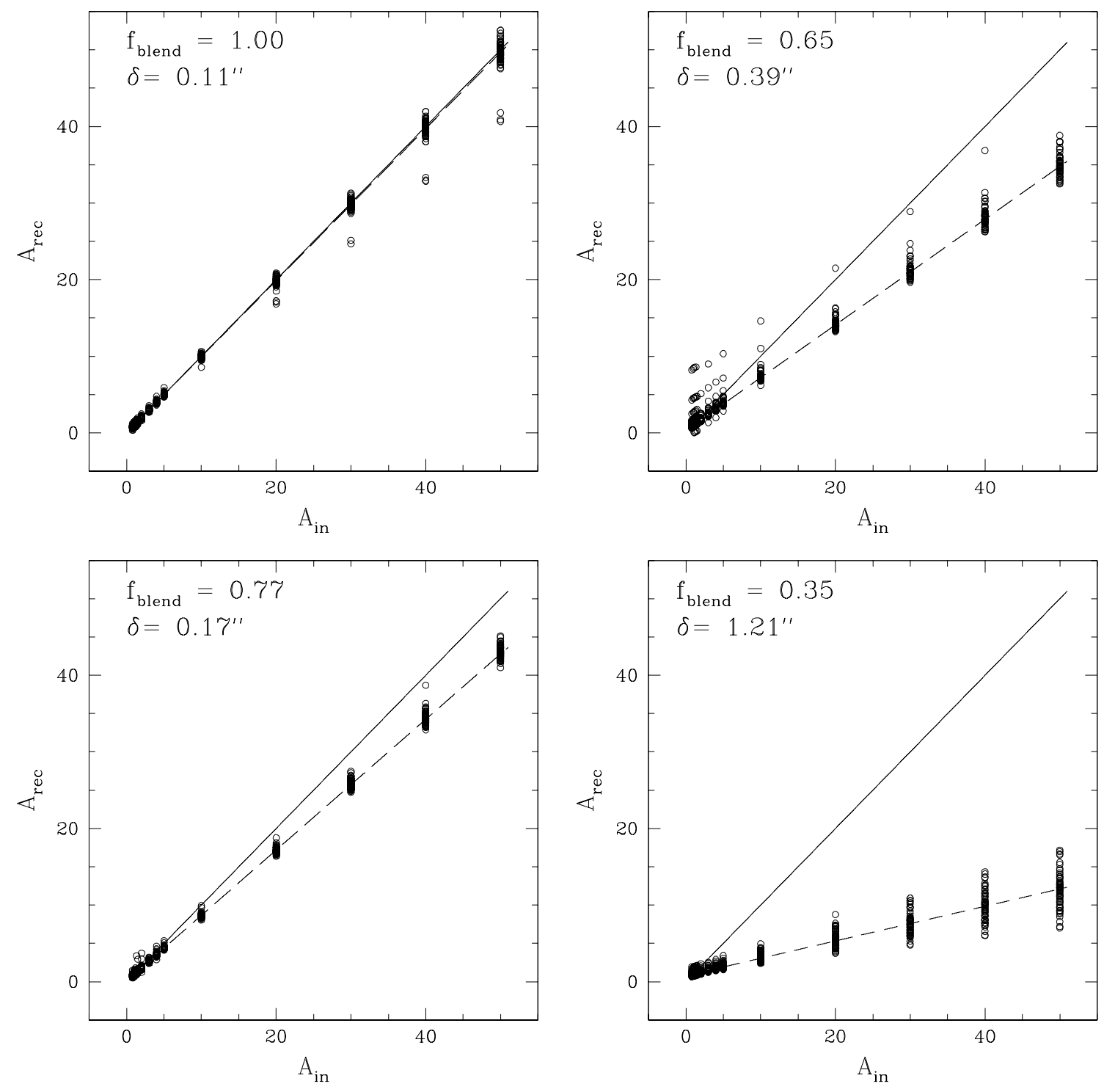

Fig. 7.- Four photometric response functions (PRFs) are plotted as recovered magnification $A^{\prime}$ versus input magnification $A$ (open circles) and compared with the "sampling efficiency" analytic response function described in $\S 3$ (solid lines). The dashed line is a least squares fit to the PRF data and in each panel the fit slope (fraction of 'lensed light' or blending fraction $\left.f_{\text {blend }}\right)$ is labeled along with the distance ( $\delta$ in arcsecs) of the 'lensed' star to the nearest photometred object. 

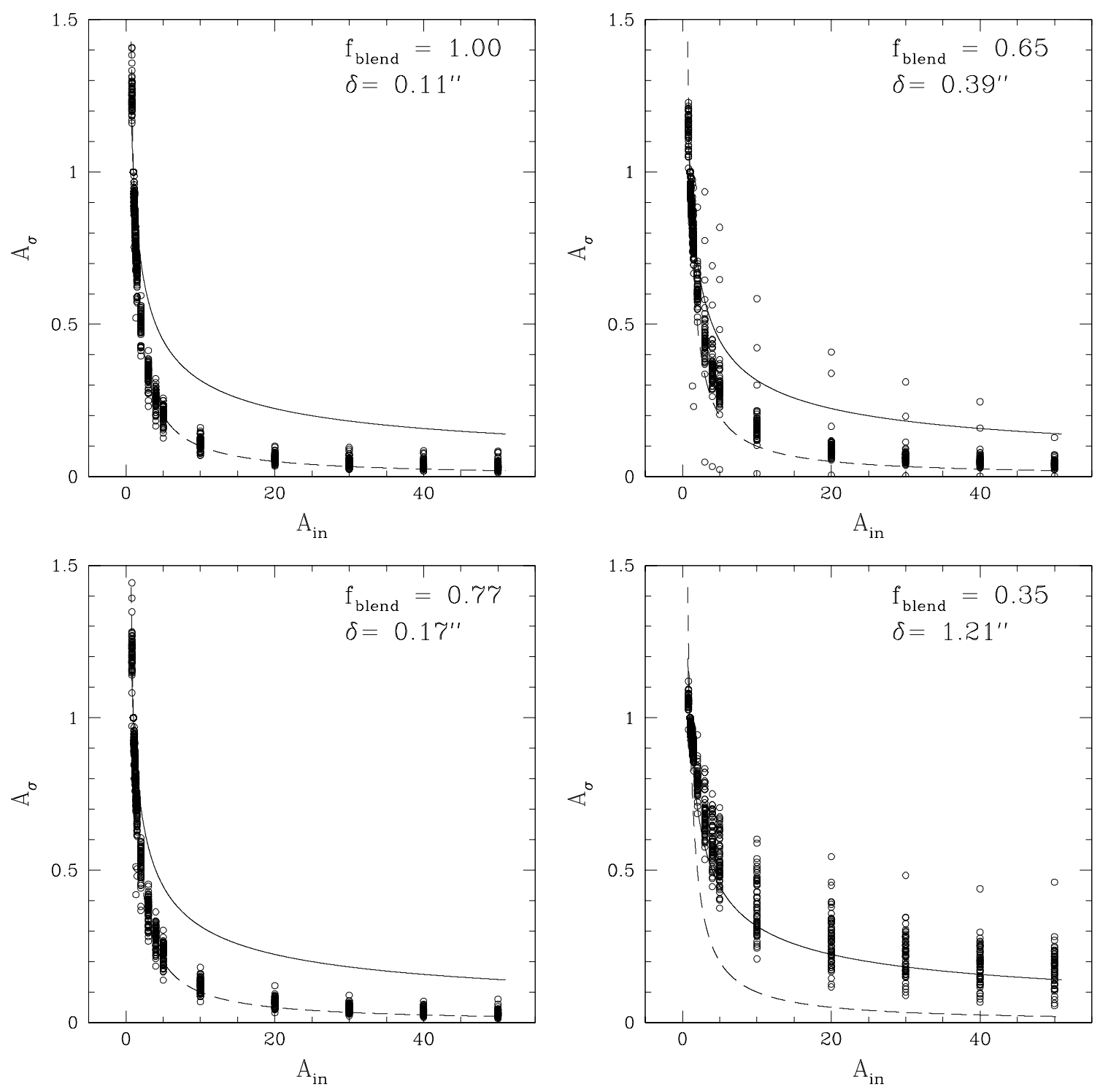

Fig. 8.- The four panels here correspond to the same PRFs shown in Figure 7 . The characteristic change in the relative error (error in magnitudes) $A_{\sigma}$ is plotted versus $A$. Overplotted on each panel is the purely Poisson response assumed in the sampling efficiency ( solid line; see $\S[$ ) and the behavior $\sim 1 / A$ (dashed line). See the text for more explanation. 


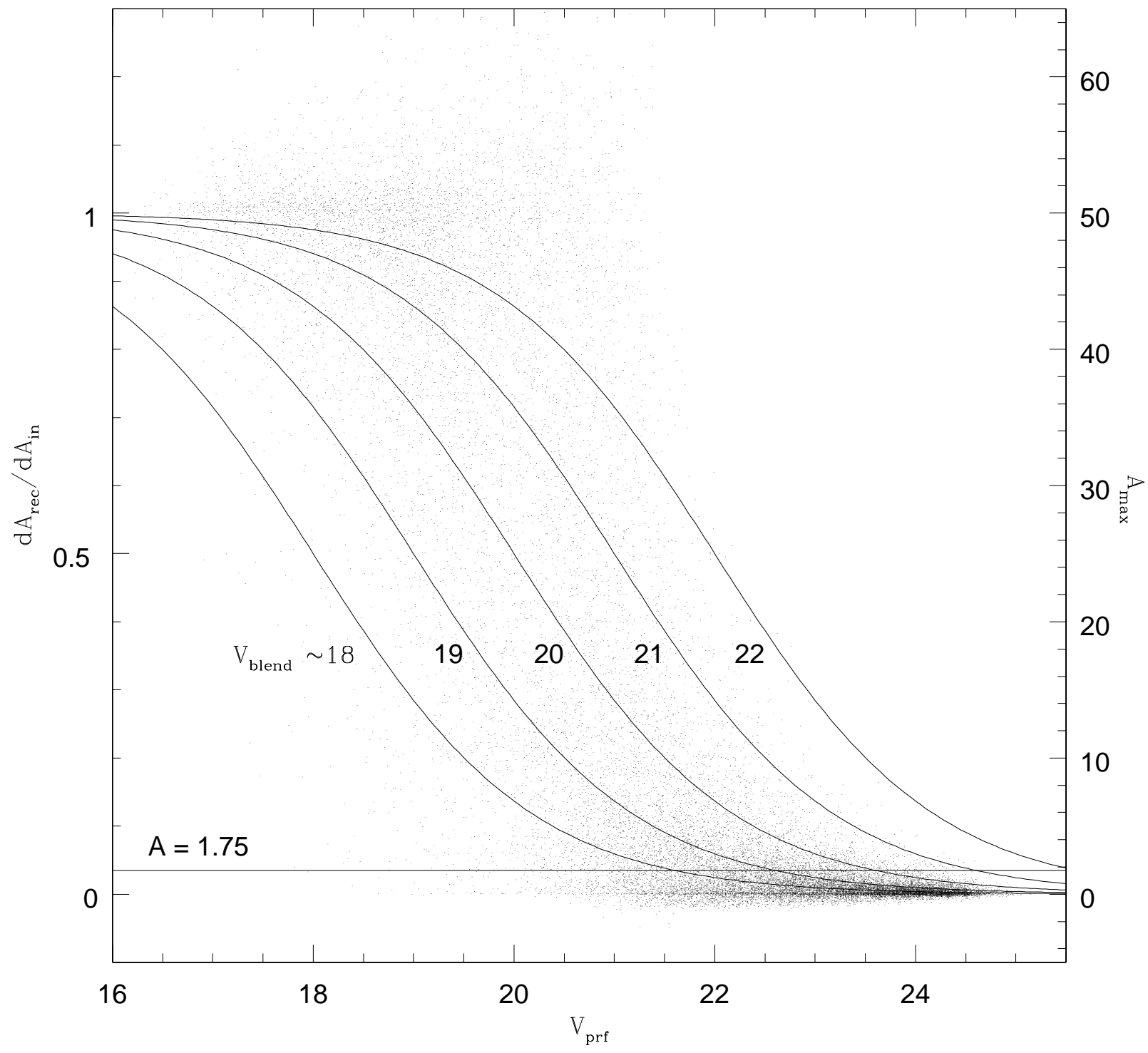

Fig. 9.- Plot of effective blend fraction (fit $m=d A_{r e c} / d A$ ) versus $V$ magnitude for the $\sim 59,000$ PRFs contained in the PRDB. Blending is not limited to faint stars, but clearly becomes worse for fainter magnitudes. Note that the distribution of PRFs shown here samples the LF in a square-root fashion (for reasons discussed in the text) and as such dim stars shown here are under-weighted. The family of curves and the right-hand axis are explained in the text. 

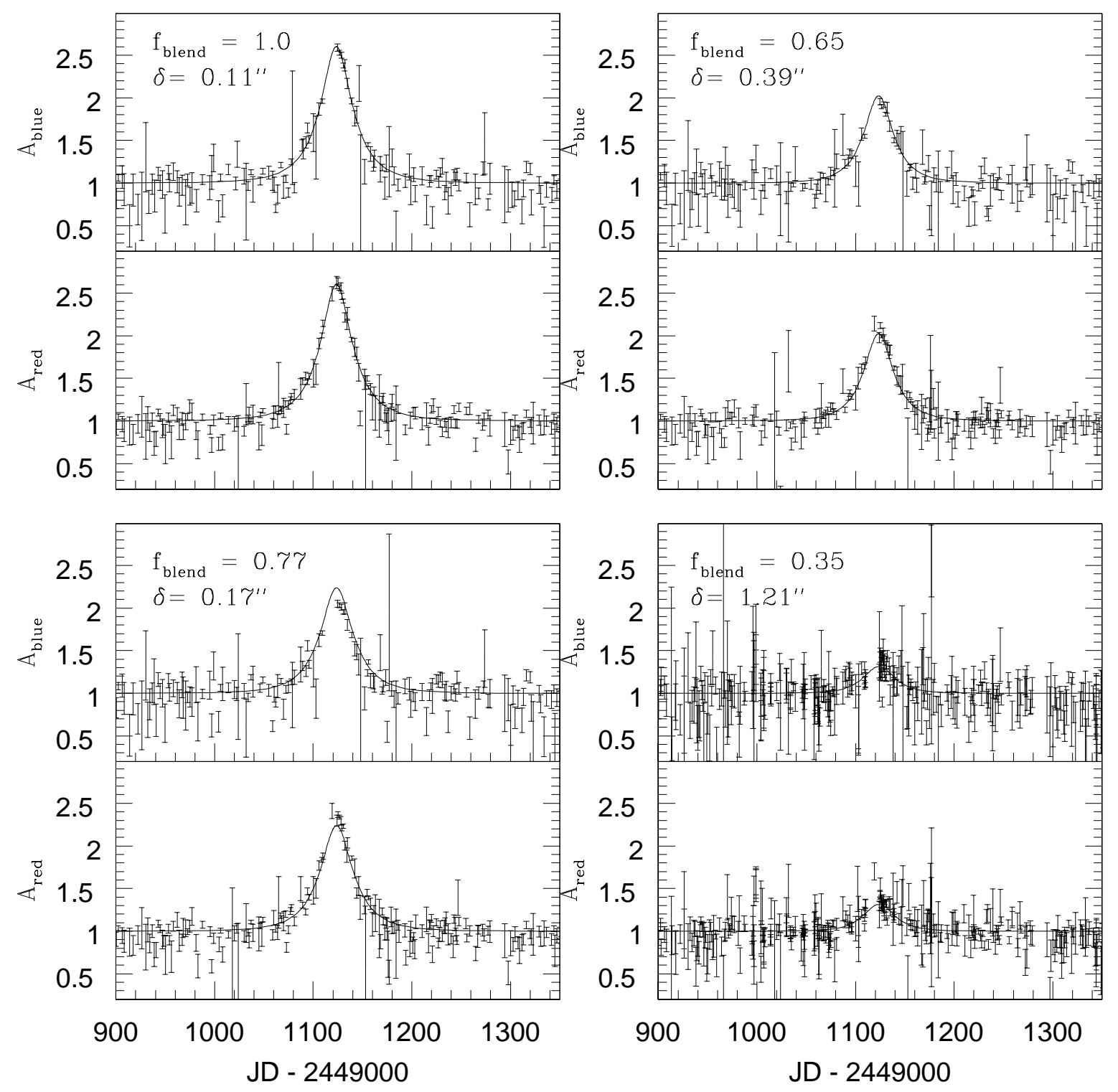

Fig. 10.- Displayed here are four example Monte Carlo lightcurves with identical input event parameters $\left(A_{\max }=\right.$ 2.2 and $\hat{t}=80$ days) generated using the same four PRFs displayed in Figures 7 and 8 . The solid line is the standard microlensing fit described in $\S$ 2. The error bars are $\pm 1 \sigma$. 


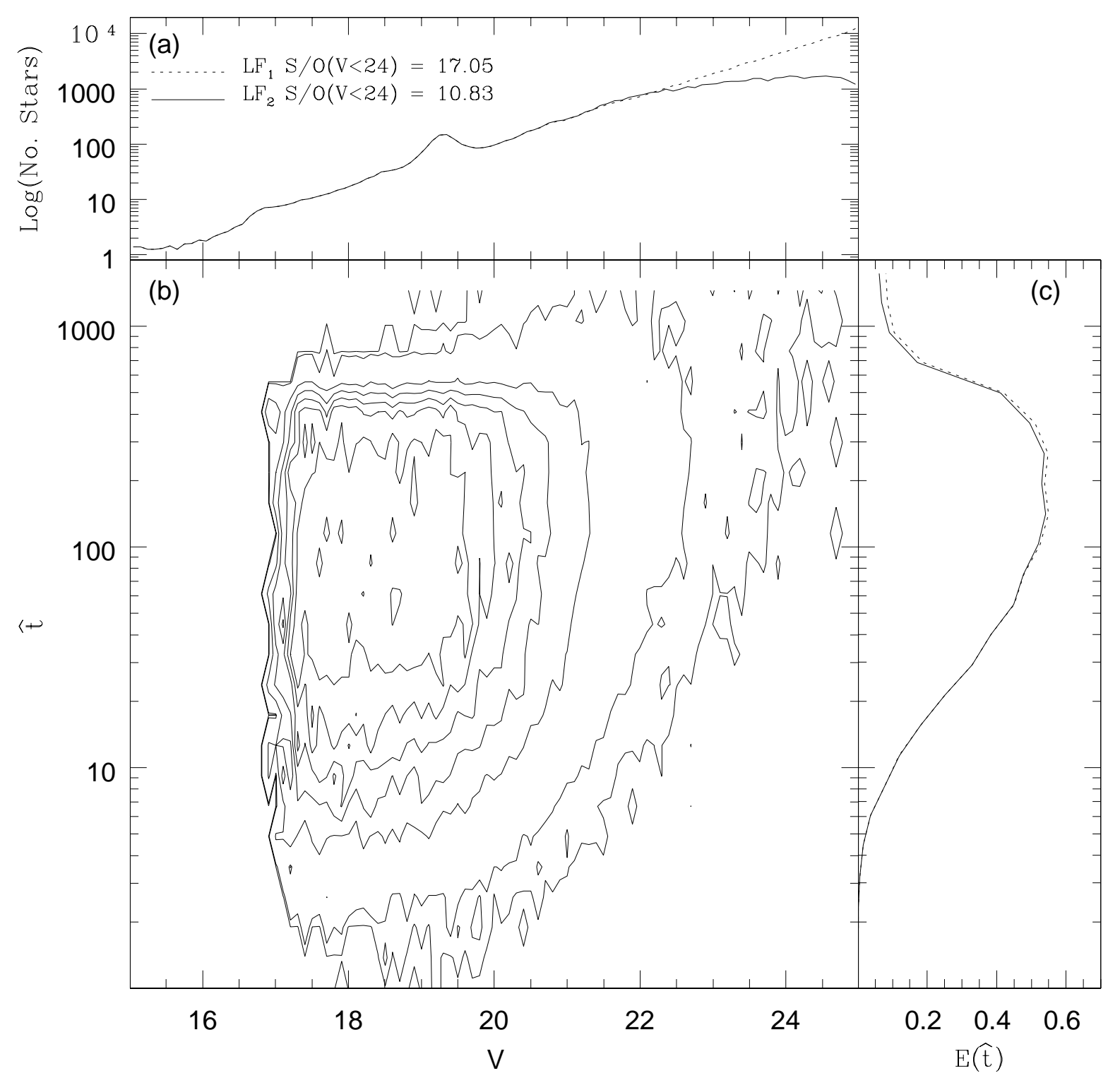

Fig. 11. - Panel (a) displays luminosity functions $L F_{1}$ (dotted line) and $L F_{2}$ (solid line), which are used to integrate $\epsilon(\hat{t}, V)$ over $V$. The function $\epsilon(\hat{t}, V)$ is shown in panel (b) as a contour plot, with the contours corresponding to efficiencies of $0.001,0.01,0.1,0.2,0.3,0.4$, and 0.5 . The function $\epsilon(\hat{t}, V)$ is described more fully in the text. The result of integrating $\epsilon(\hat{t}, V)$ over the two LFs and scaling by the appropriate normalization to obtain $\mathcal{E}(\hat{t})$ is shown in panel (c). The line coding corresponds to that in panel (a). Note that $\mathcal{E}(\hat{t})$ is fairly insensitive to the shape of the luminosity function for dim stars because, (1) the function $\epsilon(\hat{t}, V)$ is small here and (2) although the faint stars are weighted less in $L F_{2}$ they also contribute less to the normalization $S / O(V<24)$. 


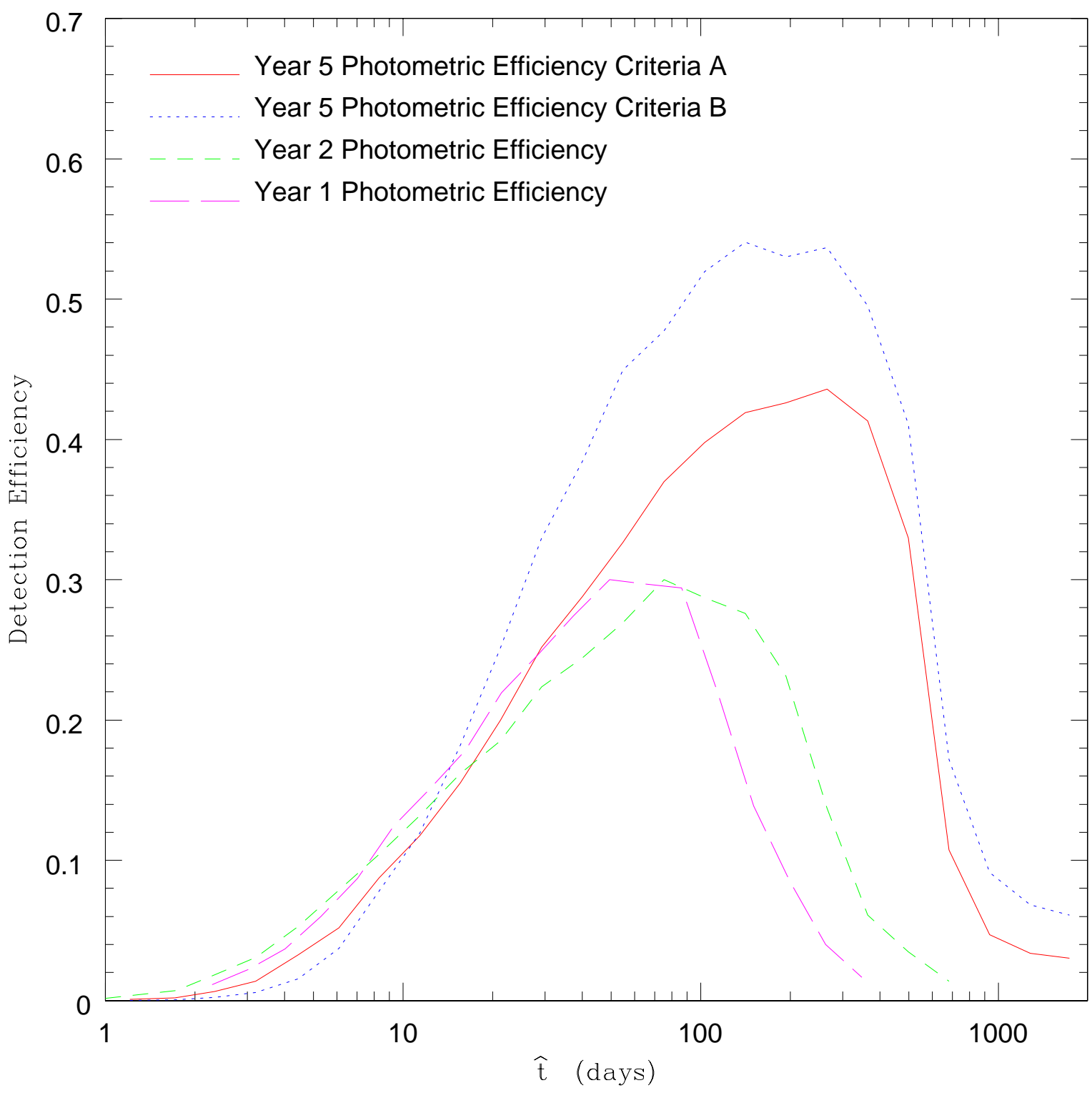

Fig. 12.- Microlensing detection efficiency (normalized to $u_{\min }<1$ ) for the 5.7-year MACHO data, as a function of event timescale $\hat{t}$. The solid line shows the 'photometric' efficiency computed for criteria set A, and the dotted line for criteria set B as described in $\S$ 5.1. For comparison the corresponding curves from A96 and A97 are also shown. 

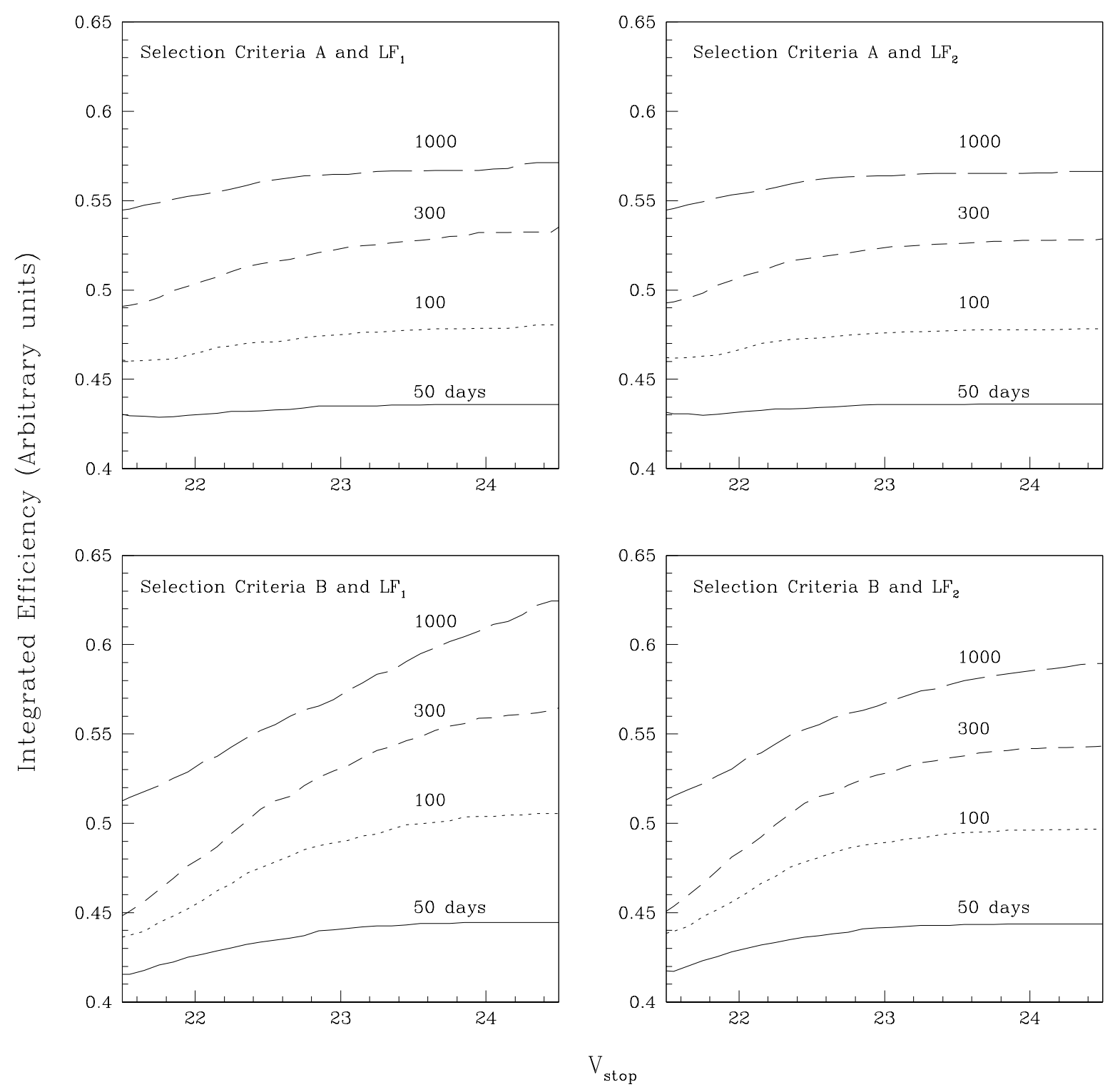

Fig. 13.- Convergence of $\mathcal{E}(\hat{t})$ with $V_{\text {stop }}$ is shown for several different durations, $\hat{t}$, and for the four combinations of selection criteria sets $\mathrm{A}$ and $\mathrm{B}$ and luminosity functions $L F_{1}$ and $L F_{2}$. 

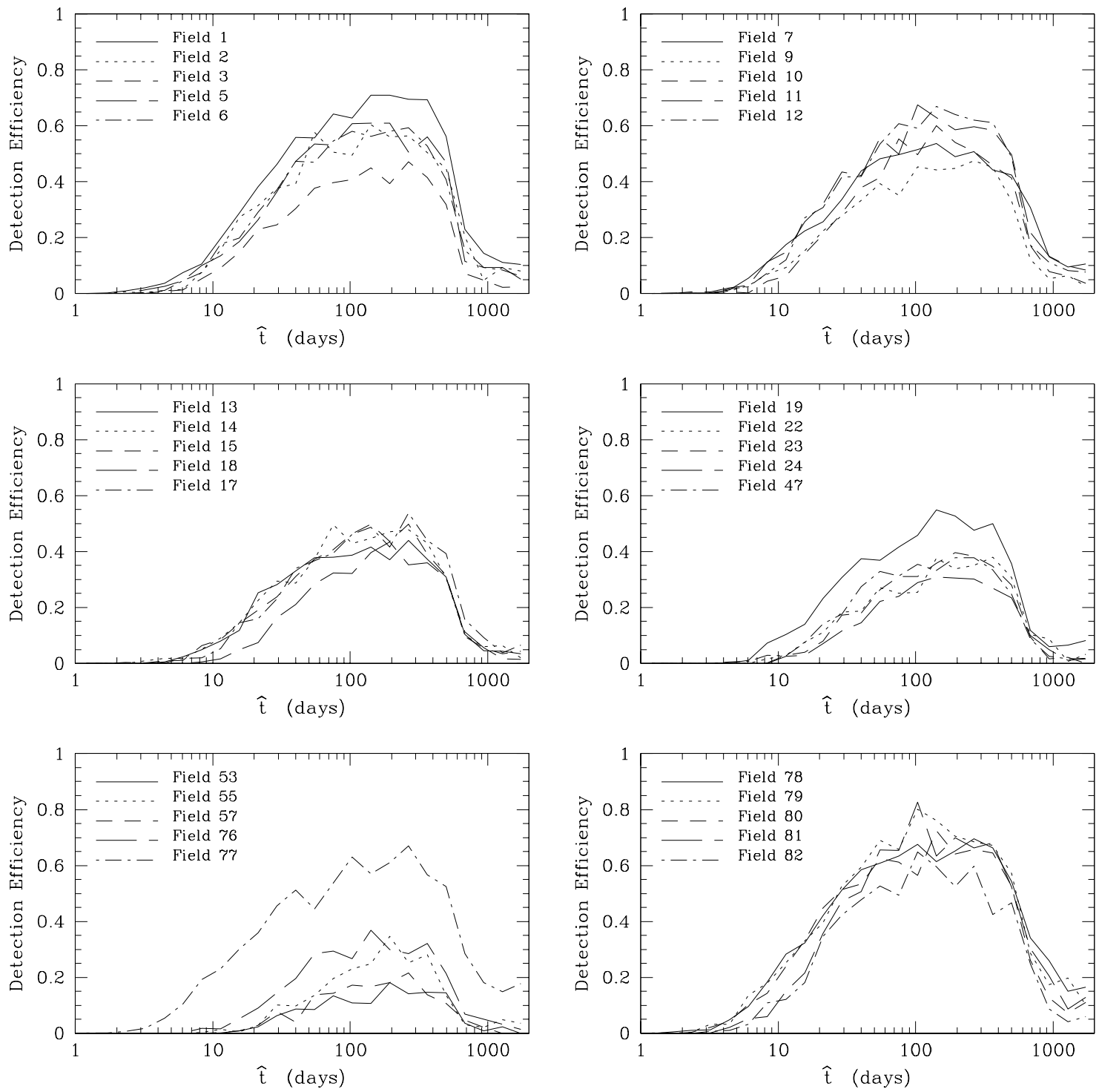

Fig. 14.- The photometric efficiency for each field is displayed for the 30 fields analyzed in A00. The different sampling rates, stellar densities, and crowding all contribute to the differences seen between fields. 

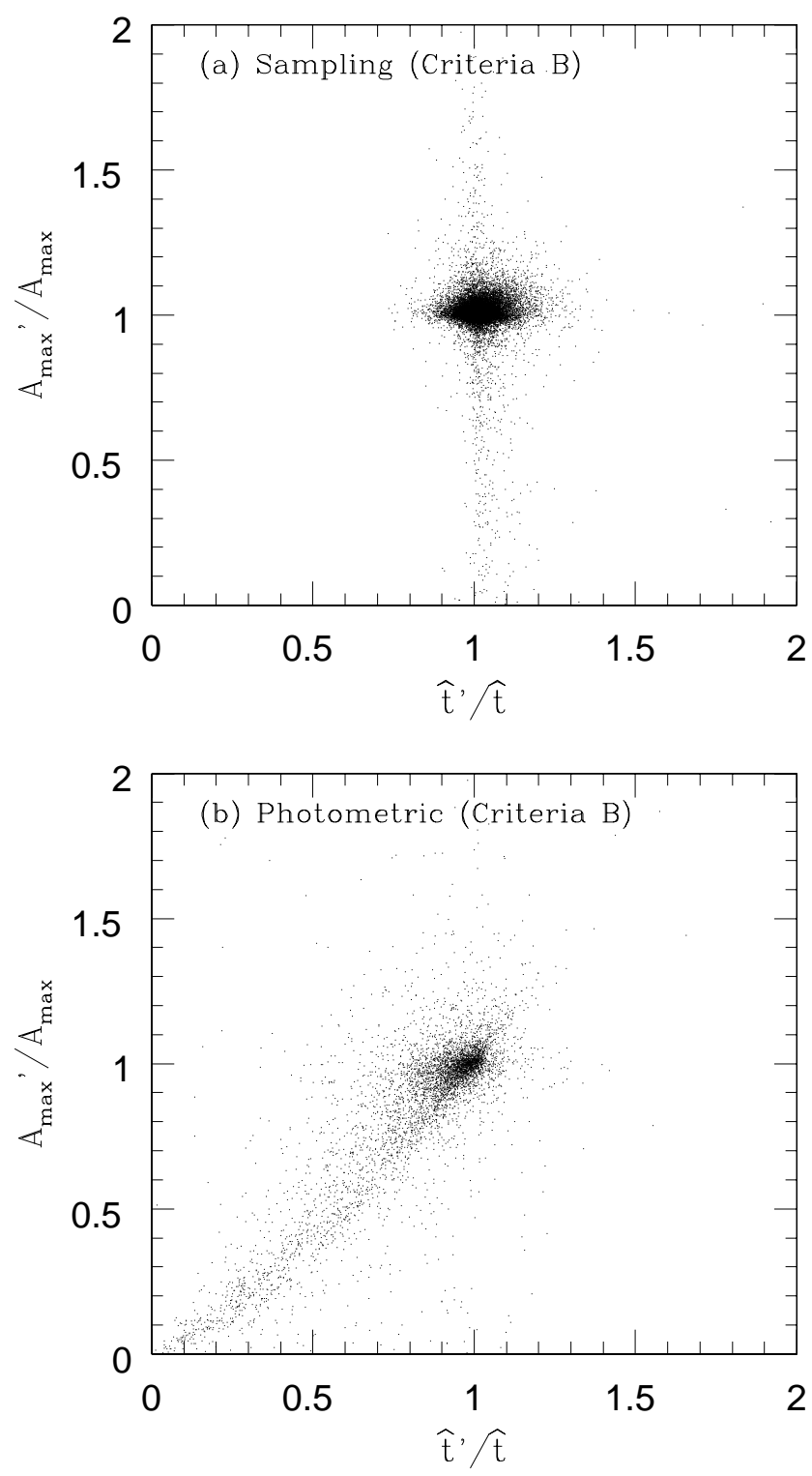

Fig. 15.- Parameter biases in the fitting code are induced by blending as illustrated here in a scatter plot of $A_{\max }^{\prime} / A_{\max }$ versus $\hat{t}^{\prime} / \hat{t}$ for a sample of Monte Carlo events. Panel (a) is a sample created with the sampling efficiency methods and panel (b) is a sample created using the photometric efficiency methods (both use selection criteria set B). 


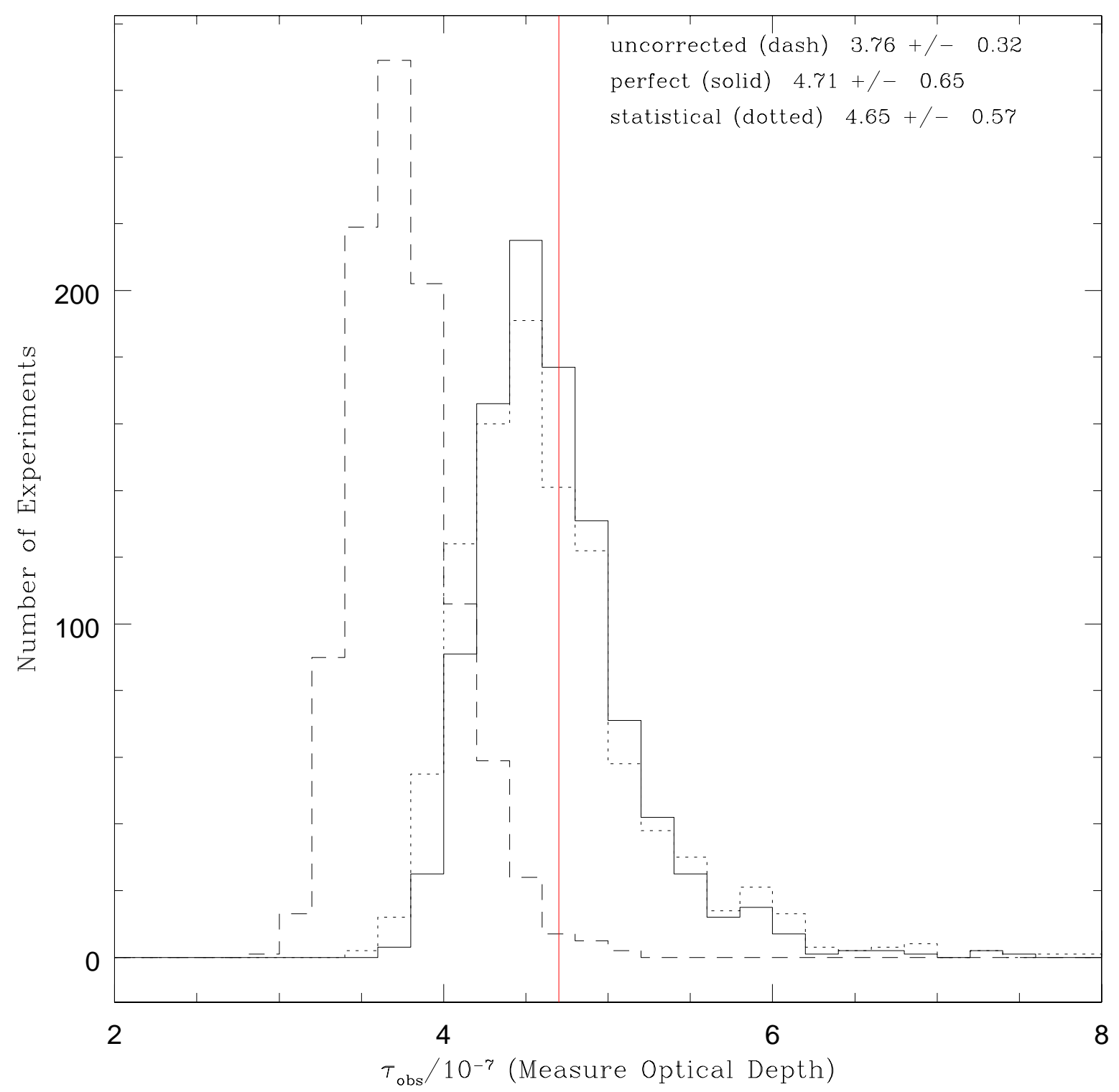

Fig. 16. - The results of a Monte Carlo $\hat{t}$ bias test described in the text is shown. Here we have used $L F_{2}$ and criteria set B along with a value of the bias correction of $\bar{\alpha}=0.785$ (Table 5 ) computed using Equation 12 . The number of expected events per trial was $N_{\text {exp }}=64$. The dashed histogram is the distribution in $\tau_{\text {meas }}$ using the uncorrected $\hat{t}^{\prime}$ to compute the optical depth, and as expected is heavily biased toward smaller optical depths. The solid histogram is the distribution using the true values of $\hat{t}$ and is exact at recovering the optical depth on average. The dotted histogram is the distribution in $\tau_{\text {meas }}$ using the statistically corrected values of $\hat{t}_{s t} \rightarrow \hat{t}^{\prime} / \bar{\alpha}$. Using $\bar{\alpha}$ has corrected for the $\hat{t}$ bias without significantly increasing the spread in optical depths. 
TABLE 1

NORMALIZATIONS

\begin{tabular}{|c|c|c|c|c|c|c|c|c|c|}
\hline Field & air & $\begin{array}{l}\text { seeing } \\
(")\end{array}$ & $\begin{array}{l}\text { sky } \\
\left(e^{-}\right)\end{array}$ & $\begin{array}{c}\text { offset } \\
\text { (V mag) }\end{array}$ & $\begin{array}{c}\text { Density } \\
\left(\text { Objs/ } \square^{\prime}\right)\end{array}$ & $\begin{array}{c}L F_{1} \\
S / O(22)\end{array}$ & $\begin{array}{c}L F_{1} \\
S / O(24)\end{array}$ & $\begin{array}{c}L F_{2} \\
S / O(22)\end{array}$ & $\begin{array}{c}L F_{2} \\
S / O(24)\end{array}$ \\
\hline 1 & 2.01 & 1.68 & 2740.0 & 0.10 & 241.3 & $3.6_{-0.7}^{+0.6}$ & $22.6_{-4.6}^{+4.0}$ & $3.7_{-0.8}^{+0.7}$ & $14.3_{-2.9}^{+2.5}$ \\
\hline 2 & 1.56 & 1.63 & 3423.0 & 0.08 & 184.7 & $2.7_{-0.5}^{+0.5}$ & $17.1_{-3.5}^{+3.1}$ & $2.8_{-0.6}^{+0.5}$ & $10.9_{-2.2}^{+1.9}$ \\
\hline 3 & 1.56 & 1.53 & 2950.0 & -0.02 & 173.4 & $1.8_{-0.3}^{+0.3}$ & $11.1_{-2.1}^{+1.8}$ & $1.8_{-0.3}^{+0.3}$ & $7.1_{-1.3}^{+1.1}$ \\
\hline 5 & 1.21 & 1.78 & 3285.0 & 0.12 & 237.9 & $2.7_{-0.6}^{+0.5}$ & $17.0_{-3.5}^{+3.2}$ & $2.8_{-0.6}^{+0.5}$ & $10.8_{-2.2}^{+2.0}$ \\
\hline 6 & 1.53 & 1.52 & 3176.0 & 0.08 & 226.4 & $2.5_{-0.5}^{+0.4}$ & $15.9_{-3.2}^{+2.8}$ & $2.6_{-0.5}^{+0.5}$ & $10.1_{-2.0}^{+1.8}$ \\
\hline 7 & 1.46 & 1.60 & 3122.0 & 0.25 & 233.7 & $2.8_{-0.6}^{+0.5}$ & $17.8_{-3.8}^{+3.3}$ & $2.9_{-0.6}^{+0.5}$ & $11.3_{-2.4}^{+2.1}$ \\
\hline 9 & 1.33 & 1.49 & 1771.0 & 0.12 & 209.7 & $1.9_{-0.4}^{+0.0}$ & $11.9_{-2.5}^{+2.0}$ & $2.0_{-0.4}^{+0.4}$ & $7.6_{-1.6}^{+1.4}$ \\
\hline 10 & 1.31 & 1.66 & 2450.0 & 0.12 & 182.3 & $2.4_{-0.5}^{+0.4}$ & $14.9_{-3.1}^{+2.7}$ & $2.4_{-0.5}^{+0.4}$ & $9.5_{-2.0}^{+1.0}$ \\
\hline 11 & 1.46 & 1.59 & 3153.0 & 0.10 & 221.9 & $2.8_{-0.6}^{+0.5}$ & $17.4_{-3.5}^{+3.0}$ & $2.8_{-0.6}^{+0.5}$ & $11.0_{-2.2}^{+1.9}$ \\
\hline 12 & 1.57 & 1.58 & 3109.0 & -0.14 & 202.9 & $2.7_{-0.5}^{-0.6}$ & $16.8_{-3.4}^{+3.0}$ & $2.8_{-0.6}^{+0.6}$ & $10.7_{-2.2}^{+1.2}$ \\
\hline 13 & 1.32 & 1.55 & 2290.0 & 0.10 & 179.6 & $\begin{array}{l}-0.5 \\
1.7_{-0.4}^{+0.3}\end{array}$ & $10.7_{-2.3}^{+1.4}$ & $1.8_{-0.4}^{+0.6}$ & $6.8_{-1.4}^{+1.2 .2}$ \\
\hline 14 & 1.32 & 1.62 & 1636.0 & 0.12 & 211.1 & $1.8_{-0.4}^{+0.4}$ & $11.3_{-2.2}^{+1.3}$ & $1.9_{-0.4}^{+0.3}$ & $7.2_{-1.4}^{+1.2}$ \\
\hline 15 & 1.32 & 1.80 & 1543.0 & -0.08 & 182.9 & $1.9_{-0.4}^{-0.4}$ & $12.2_{-2.2}^{+2.2}$ & $\begin{array}{l}1.0-0.4 \\
2.0_{-0.4}^{+0.4}\end{array}$ & $7.8_{-1.6}^{+1.4}$ \\
\hline 17 & 1.36 & 1.55 & 2250.0 & 0.15 & 176.9 & $2.3_{-0.4}^{+0.4}$ & $14.2_{-2.7}^{+2.3}$ & $2.3_{-0.4}^{+0.4}$ & $9.0_{-1.7}^{+1.5}$ \\
\hline 18 & 1.34 & 1.51 & 2310.0 & 0.15 & 214.0 & $2.1_{-0.4}^{+0.4}$ & $13.3_{-2.7}^{+2.3}$ & $2.2_{-0.4}^{+0.4}$ & $8.5_{-1.7}^{+1.5}$ \\
\hline 19 & 2.50 & 1.80 & 2951.0 & 0.17 & 169.6 & $2.5_{-0.5}^{+0.5}$ & $\begin{array}{r}15.8_{-3.2}^{+2.9} \\
\end{array}$ & $2.6_{-0.5}^{+0.5}$ & $10.1_{-2.0}^{+1.8}$ \\
\hline 22 & 1.38 & 1.71 & 2250.0 & 0.17 & 141.1 & $1.7_{-0.3}^{+0.3}$ & $\begin{array}{l}10.7_{-2.2}^{+1.9} \\
\end{array}$ & $1.8_{-0.4}^{+0.3}$ & $6.8_{-1.4}^{+1.2}$ \\
\hline 23 & 1.38 & 1.65 & 2280.0 & 0.19 & 158.4 & $2.1_{-0.4}^{+0.4}$ & $13.1_{-2.5}^{+2.2}$ & $2.2_{-0.4}^{+0.4}$ & $8.3_{-1.6}^{+1.4}$ \\
\hline 24 & 1.35 & 2.00 & 2090.0 & 0.25 & 148.3 & $1.7_{-0.3}^{+0.3}$ & $10.5_{-2.1}^{+1.9}$ & $1.7_{-0.4}^{+0.3}$ & $6.7_{-1.4}^{+1.2}$ \\
\hline 47 & 1.60 & 1.57 & 2440.0 & 0.15 & 141.7 & $1.5_{-0.3}^{+0.3}$ & $9.7_{-2.0}^{+1.1}$ & $1.6_{-0.3}^{+0.3}$ & $6.2_{-1.3}^{+1.1}$ \\
\hline 53 & 2.14 & 1.79 & 2840.0 & 0.40 & 100.0 & $1.6_{-0.3}^{+0.3}$ & $10.0_{-2.0}^{+1.9}$ & $1.6_{-0.3}^{+0.3}$ & $6.4_{-1.3}^{+1.2}$ \\
\hline 55 & 2.01 & 1.83 & 2590.0 & 0.23 & 93.0 & $1.9_{-0.4}^{+0.3}$ & $11.7_{-2.4}^{+2.0}$ & $1.9_{-0.4}^{+0.4}$ & $7.4_{-1.5}^{+1.4}$ \\
\hline 57 & 1.84 & 1.89 & 2510.0 & 0.29 & 98.9 & $1.6_{-0.3}^{+0.4}$ & $\begin{array}{c}-2.4 \\
9.8_{-2.0}^{+1.8}\end{array}$ & $1.6_{-0.3}^{+0.3}$ & $6.2_{-1.2}^{+1.1}$ \\
\hline 76 & 1.25 & 1.60 & 2470.0 & 0.15 & 167.0 & $\begin{array}{l}-0.3 \\
1.8_{-0.4}^{+0.3}\end{array}$ & $\begin{array}{c}-2.0 \\
11.1_{-2.2}^{+2.0}\end{array}$ & $1.8_{-0.4}^{+0.33}$ & $7.1_{-1.4}^{+1.2}$ \\
\hline 77 & 1.39 & 1.48 & 2314.0 & 0.06 & 316.3 & $4.4_{-0.9}^{+0.4}$ & $27.9_{-5.8}^{+5.2}$ & $4.6_{-1.0}^{+0.8}$ & $17.8_{-3.7}^{+3.4}$ \\
\hline 78 & 1.41 & 1.58 & 3545.0 & 0.12 & 290.2 & $4.6_{-1.0}^{+0.8}$ & $28.8_{-6.0}^{+5.8}$ & $4.7_{-1.0}^{+0.9}$ & $18.3_{-3.8}^{+3.3}$ \\
\hline 79 & 1.43 & 1.65 & 3465.0 & 0.08 & 255.5 & $4.3_{-0.9}^{+0.0}$ & $26.7_{-5.5}^{+4.0}$ & $\begin{array}{r}-1.0 \\
4.4_{-0.9}^{+0.8}\end{array}$ & $17.0_{-3.5}^{+3.8}$ \\
\hline 80 & 1.43 & 1.56 & 3574.0 & 0.00 & 237.9 & $3.4_{-0.7}^{+0.6}$ & $21.6_{-4.4}^{+3.5}$ & $3.5_{-0.7}^{+0.6}$ & $13.7_{-2.8}^{+2.5}$ \\
\hline 81 & 1.52 & 1.70 & 3657.0 & -0.04 & 210.6 & $4.0_{-0.8}^{+0.7}$ & $25.2_{-5.1}^{+4.4}$ & $4.1_{-0.8}^{+0.7}$ & $16.0_{-3.2}^{+2.8}$ \\
\hline 82 & 1.52 & 1.45 & 3580.0 & -0.04 & 170.4 & $3.0_{-0.6}^{+0.5}$ & $18.9_{-3.6}^{+3.1}$ & $3.1_{-0.6}^{+0.5}$ & $12.0_{-2.3}^{+2.0}$ \\
\hline
\end{tabular}

The table lists the normalizations (columns 7-10) for each of the 30 fields, along with conservative estimated errors. The columns 2-4 list the airmass, seeing (in arcsecs), and sky level (in electrons) for the MACHO template images. Column 5 is the offset for each field used in $\S 4.1 .2$. Column 6 is the average density of each field in Objects/ $\square^{\prime}$. 
TABLE 2

PDRB CHUNKS

\begin{tabular}{rrrccccc}
\hline \hline ID & Field & Chunk & Template & Objs/口' & $N_{\text {cond }}$ & $N_{P R F} /$ grid & $N_{P R F}$ \\
\hline 1 & 2 & 9 & 5672 & 143.9 & 67 & 64 & 3226 \\
2 & 7 & 40 & 5668 & 196.4 & 66 & 81 & 4209 \\
3 & 19 & 22 & 4562 & 142.1 & 72 & 64 & 3219 \\
4 & 22 & 38 & 1696 & 93.7 & 64 & 64 & 3419 \\
5 & 55 & 54 & 5311 & 98.1 & 69 & 64 & 3294 \\
6 & 77 & 27 & 4203 & 257.4 & 67 & 144 & 6884 \\
7 & 77 & 50 & 2962 & 308.9 & 66 & 156 & 7622 \\
8 & 78 & 6 & 5663 & 318.7 & 69 & 156 & 8381 \\
9 & 78 & 47 & 5663 & 269.5 & 77 & 144 & 8074 \\
10 & 81 & 18 & 5673 & 202.9 & 72 & 156 & 6653 \\
\hline
\end{tabular}

The ten $5^{\prime} \times 5^{\prime}$ chunks used to create the PRDB span the observed range of density $\left(\operatorname{Objs} / \square^{\prime}\right)$ and are widely spaced over the face of the LMC. For reference we give the template observation number, the number of observing conditions $\left(N_{\text {cond }}\right)$ used for each chunk, the number of artificial stars per grid $\left(N_{P R F} /\right.$ grid $)$ added to each chunk (60 grids per chunk) and the number of useful PRFs generated $\left(N_{P R F}\right)$ from each chunk. 
TABLE 3

EfFiciency TABLE

\begin{tabular}{cccccc}
\hline \hline Bin & $\hat{t}_{\text {min }}$ & $\hat{t}_{\text {max }}$ & $\hat{t}$ & $\begin{array}{c}\text { Criteria set A } \\
\mathcal{E}(\hat{t})\end{array}$ & $\begin{array}{c}\text { Criteria set B } \\
\mathcal{E}(\hat{t})\end{array}$ \\
\hline 1 & 1.00 & 1.46 & 1.20 & $8.7 \mathrm{e}-04$ & $1.9 \mathrm{e}-04$ \\
2 & 1.46 & 2.00 & 1.70 & $2.1 \mathrm{e}-03$ & $5.7 \mathrm{e}-04$ \\
3 & 2.00 & 2.70 & 2.32 & $6.5 \mathrm{e}-03$ & $2.2 \mathrm{e}-03$ \\
4 & 2.70 & 3.80 & 3.20 & $1.3 \mathrm{e}-02$ & $5.7 \mathrm{e}-03$ \\
5 & 3.80 & 5.20 & 4.44 & $3.2 \mathrm{e}-02$ & $1.5 \mathrm{e}-02$ \\
6 & 5.20 & 7.10 & 6.07 & $5.2 \mathrm{e}-02$ & $3.7 \mathrm{e}-02$ \\
7 & 7.10 & 9.70 & 8.29 & $8.7 \mathrm{e}-02$ & $7.8 \mathrm{e}-02$ \\
8 & 9.70 & 13.30 & 11.35 & $1.1 \mathrm{e}-01$ & $1.1 \mathrm{e}-01$ \\
9 & 13.30 & 18.20 & 15.55 & $1.5 \mathrm{e}-01$ & $1.8 \mathrm{e}-01$ \\
10 & 18.20 & 24.90 & 21.28 & $1.9 \mathrm{e}-01$ & $2.5 \mathrm{e}-01$ \\
11 & 24.90 & 34.20 & 29.18 & $2.5 \mathrm{e}-01$ & $3.2 \mathrm{e}-01$ \\
12 & 34.20 & 46.80 & 40.00 & $2.8 \mathrm{e}-01$ & $3.8 \mathrm{e}-01$ \\
13 & 46.80 & 64.20 & 54.81 & $3.2 \mathrm{e}-01$ & $4.4 \mathrm{e}-01$ \\
14 & 64.20 & 88.00 & 75.16 & $3.6 \mathrm{e}-01$ & $4.7 \mathrm{e}-01$ \\
15 & 88.00 & 121.00 & 103.18 & $3.9 \mathrm{e}-01$ & $5.1 \mathrm{e}-01$ \\
16 & 121.00 & 165.00 & 141.29 & $4.1 \mathrm{e}-01$ & $5.4 \mathrm{e}-01$ \\
17 & 165.00 & 227.00 & 193.53 & $4.2 \mathrm{e}-01$ & $5.3 \mathrm{e}-01$ \\
18 & 227.00 & 311.00 & 265.70 & $4.3 \mathrm{e}-01$ & $5.3 \mathrm{e}-01$ \\
19 & 311.00 & 426.00 & 363.98 & $4.1 \mathrm{e}-01$ & $4.9 \mathrm{e}-01$ \\
20 & 426.00 & 584.00 & 498.78 & $3.2 \mathrm{e}-01$ & $4.1 \mathrm{e}-01$ \\
21 & 584.00 & 800.00 & 683.52 & $1.0 \mathrm{e}-01$ & $1.7 \mathrm{e}-01$ \\
22 & 800.00 & 1096.00 & 936.37 & $4.7 \mathrm{e}-02$ & $9.1 \mathrm{e}-02$ \\
23 & 1096.00 & 1502.00 & 1283.04 & $3.3 \mathrm{e}-02$ & $6.8 \mathrm{e}-02$ \\
24 & 1502.00 & 2000.00 & 1733.14 & $3.0 \mathrm{e}-02$ & $6.1 \mathrm{e}-02$ \\
\hline
\end{tabular}

Efficiencies presented in A00 and also shown in Figure 12 for selection criteria set $\mathrm{A}$ and $\mathrm{B}$. There are 24 bins in duration with $\hat{t}_{\min }$ and $\hat{t}_{\max }$ delineating each bin. The quantity $\hat{t}$ marks the logarithmic center of each bin. 
TABLE 4

ERror Budget

\begin{tabular}{clrl}
\hline \hline Number & Sign & \multicolumn{1}{c}{ Size } & \multicolumn{1}{c}{ Note } \\
\hline 1 & $?$ & $\sim 20 \%$ & LF normalization/exposure \\
2 & $?$ & $\sim 1-3 \%$ & LF for faint stars $V \gtrsim 22.5$ \\
3 & $?$ & $\lesssim 1 \%$ & Finite number of Monte Carlo events \\
4 & + & $\sim 6-8 \%$ & Overcompleteness bias \\
5 & - & $\sim 3 \%$ & 1st neighbor PRF bias \\
6 & + & $\sim 1 \%$ & LF in outer 'disk' fields \\
7 & - & $\lesssim 1 \%$ & Convergence for $\hat{t}>100$ days \\
8 & $?$ & $? \%$ & Binary stars in LF \\
9 & $?$ & $? \%$ & No exotic lensing events \\
\hline
\end{tabular}

This table details our error budget for the efficiency. Column 2 gives the probable direction, if known, in which the error might propagate to the efficiency, e. g., \#4 could bias the efficiency to higher values, causing the optical depth to be slightly underestimated. Two possible corrections that are not estimated here are \#8 and \#9 (see the text).

TABLE 5

$\hat{t}$ Bias CORrections

\begin{tabular}{ccccc}
\hline \hline $\begin{array}{c}\text { Selection } \\
\text { Criteria }\end{array}$ & LF & \multicolumn{3}{c}{ Median $\bar{\alpha}$} \\
\hline A & 1 & $0.1 M_{\odot}$ & $0.5 M_{\odot}$ & $1.0 M_{\odot}$ \\
A & 2 & 0.867 & 0.826 & 0.812 \\
B & 1 & 0.826 & 0.777 & 0.818 \\
B & 2 & 0.828 & 0.785 & 0.759 \\
\hline
\end{tabular}

This table lists $\hat{t}$ bias corrections which may be used to statistically correct the unblended fit $\hat{t}$ 's. This statistical correction is a function of the selection criteria set used, but also sensitive to the average duration of the observed microlensing events, here delineated using a standard Galactic halo model (model S) with delta-function masses of $0.1,0.5$, and $1.0 M_{\odot}$, corresponding to mean durations of 41,92 , and 130 days, respectively. We recommend using $L F_{2}$ and $0.5 M_{\odot}$. 